\title{
Cyclic cocycles in the spectral action
}

\author{
Teun D.H. van Nuland, Walter D. van Suijlekom ${ }^{+}$ \\ Radboud University, Heyendaalseweg 135, \\ 6525 AJ Nijmegen, The Netherlands
}

September 19, 2022

\begin{abstract}
We show that the spectral action, when perturbed by a gauge potential, can be written as a series of Chern-Simons actions and Yang-Mills actions of all orders. In the odd orders, generalized Chern-Simons forms are integrated against an odd $(b, B)$-cocycle, whereas, in the even orders, powers of the curvature are integrated against $(b, B)$-cocycles that are Hochschild cocycles as well. In both cases, the Hochschild cochains are derived from the Taylor series expansion of the spectral action $\operatorname{Tr}(f(D+V))$ in powers of $V=\pi_{D}(A)$, but unlike the Taylor expansion we expand in increasing order of the forms in $A$. This extends [11], which computes only the scale-invariant part of the spectral action, works in dimension at most 4 , and assumes the vanishing tadpole hypothesis. In our situation, we obtain a truly infinite odd $(b, B)$-cocycle. The analysis involved draws from recent results in multiple operator integration, which also allows us to give conditions under which this cocycle is entire, and under which our expansion is absolutely convergent. As a consequence of our expansion and of the gauge invariance of the spectral action, we show that the odd $(b, B)$-cocycle pairs trivially with $K_{1}$.
\end{abstract}

\section{Contents}

1 Introduction

2 Preliminaries on multiple operator integrals

3 Estimating the multiple operator integral in the $s$-summable case

3.1 Bound on the multiple operator integral . . . . . . . . . . . . . 9

3.2 Continuity of the multiple operator integral . . . . . . . . . . . . . . . . 11

4 Cyclic cocycles and universal forms underlying the spectral action 11

4.1 Hochschild and cyclic cocycles . . . . . . . . . . . . . . . . 13

4.2 Cyclic cocycles associated to multiple operator integrals . . . . . . . . . . . . . 14

4.3 Derivatives of the spectral action in terms of universal forms . . . . . . . . . . 15

4.4 Near-tracial behavior of $\int_{\phi} \ldots \ldots \ldots \ldots \ldots \ldots \ldots \ldots$

4.5 Higher-order generalized Chern-Simons forms . . . . . . . . . . . . . 18

*t.vannuland@math.ru.nl

†waltervs@math.ru.nl 
5 Expansion of the spectral action in terms of $(b, B)$-cocycles

5.1 Truncated expansion . . . . . . . . . . . . . . . . . . . 19

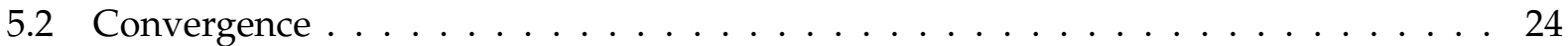

6 Gauge invariance and the pairing with K-theory

\section{Introduction}

The spectral action [2, 3] is one of the key instruments in the applications of noncommutative geometry to particle physics. With inner fluctuations [10] of a noncommutative manifold playing the role of gauge potentials, the spectral action principle yields Lagrangians for them. In fact, the asymptotic behavior of the spectral action for small momenta leads in this way to local field theories. For example, this provides the simplest way known to geometrically explain the dynamics and interactions of the gauge bosons and the Higgs boson in the Standard Model Lagrangian as an effective field theory [4] (see also the textbooks [13, 25]). These techniques extend to more general noncommutative manifolds, and, indeed, if one restrict to the scale-invariant part, one may naturally identify a Yang-Mills term and a Chern-Simons term to elegantly appear in the spectral action [11]. However, it remains an open problem to determine the general form of the spectral action without resorting to the scale-invariant part, even though from the physical perspective there is a strong desire to go beyond this effective field theory approach.

Motivated by this, we study the spectral action when it is expanded in terms of inner fluctuations associated to an arbitrary noncommutative manifold, without resorting to heat-kernel techniques. Indeed, the latter are not always available and an understanding of the full spectral action could provide deeper insight in the origin of these gauge theories from noncommutative geometry. Let us now give a more precise description of our setup.

We let $(\mathcal{A}, \mathcal{H}, D)$ be an $s$-summable spectral triple ( $c f$. Definition 12 below). If $f: \mathbb{R} \rightarrow \mathbb{C}$ is a suitably nice function we may define the spectral action [3]:

$$
\operatorname{Tr}(f(D)) \text {. }
$$

An inner fluctuation, as explained in [10], is given by a Hermitian universal one-form

$$
A=\sum_{j=1}^{n} a_{j} d b_{j} \in \Omega^{1}(\mathcal{A})
$$

for elements $a_{j}, b_{j} \in \mathcal{A}$. The terminology 'fluctuation' comes from representing $A$ on $\mathcal{H}$ as

$$
V:=\pi_{D}(A):=\sum_{j=1}^{n} a_{j}\left[D, b_{j}\right] \in \mathcal{B}(\mathcal{H})_{\mathrm{sa}}
$$

and fluctuating $D$ to $D+V$ in the spectral action. The variation of the spectral action under the inner fluctuation is then given by

$$
\operatorname{Tr}(f(D+V))-\operatorname{Tr}(f(D)) .
$$

As spectral triples can be understood as noncommutative spin manifolds encoding the gauge fields as an inner structure, one could hope that perturbations of the spectral action could be understood in terms of noncommutative versions of geometrical, gauge theoretical concepts. Hence 
we would like to express (3) in terms of universal forms constructed from $A$. To express an action functional in terms of universal forms, one is naturally led to cyclic cohomology. As it turns out, hidden inside the spectral action we will identify an odd $(b, B)$-cocycle $\left(\tilde{\psi}_{1}, \tilde{\psi}_{3}, \ldots\right)$ and an even $(b, B)$-cocycle $\left(\phi_{2}, \phi_{4}, \ldots\right)$ for which $b \phi_{2 k}=B \phi_{2 k}=0$, i.e., each Hochschild cochain $\phi_{2 k}$ forms its own $(b, B)$-cocycle $\left(0, \ldots, 0, \phi_{2 k}, 0, \ldots\right)$. On the other hand, the odd $(b, B)$-cocycle $\left(\tilde{\psi}_{2 k+1}\right)$ is truly infinite.

The main result of this paper is that for suitable $f: \mathbb{R} \rightarrow \mathbb{C}$ we may expand

$$
\operatorname{Tr}(f(D+V)-f(D))=\sum_{k=1}^{\infty}\left(\int_{\psi_{2 k-1}} \operatorname{cs}_{2 k-1}(A)+\frac{1}{2 k} \int_{\phi_{2 k}} F^{k}\right),
$$

in which the series converges absolutely. Here $\psi_{2 k-1}$ is a scalar multiple of $\tilde{\psi}_{2 k-1}, F_{t}=t d A+t^{2} A^{2}$, so that $F=F_{1}$ is the curvature of $A$, and $\operatorname{cs}_{2 k-1}(A)=\int_{0}^{1} A F_{t}^{k-1} d t$ is a generalized noncommutative Chern-Simons form. We also give a bound on the remainder of this expansion.

As already mentioned, a similar result was shown earlier to hold for the scale-invariant part of the spectral action. Indeed, Connes and Chamseddine [11] expressed the scale-invariant part in dimension $\leq 4$ as

$$
\zeta_{D+V}(0)-\zeta_{D}(0)=-\frac{1}{4} \int_{\tau_{0}}\left(d A+A^{2}\right)+\frac{1}{2} \int_{\psi}\left(A d A+\frac{2}{3} A^{3}\right)
$$

for a certain Hochschild 4-cocycle $\tau_{0}$ and cyclic 3-cocycle $\psi$.

Interestingly, a key role in our extension of this result to the full spectral action will be played by multiple operator integrals. It is the natural replacement of residues in this context, and also allows to go beyond dimension 4 . For our analysis of the cocycle structure that appears in the full spectral action we take the Taylor series expansion as a starting point. This explains the appearance of multiple operator integrals, as traces thereof are multilinear extensions of the derivatives of the spectral action. This viewpoint is also studied in [22, 24], where multiple operator integrals are used to investigate the Taylor expansion of the spectral action. As we will show, multiple operator integrals can also be used to define cyclic cocycles, because of some known properties of the multiple operator integral that have been proved in increasing generality in the last decades (e.g., in [1, 12, 17, 22, 24]). We push these results a bit further, in Section 3, by proving estimates and continuity properties for the multiple operator integral when the self-adjoint operator has an s-summable resolvent. Extending these results to the case of non-unital spectral triples is left open for future research.

These techniques also allow us to show that the found $(b, B)$-cocycles are entire in the sense of [8]. This makes it meaningful to analyze their pairing with K-theory, which we find to be trivial in Section 6 ,

\section{Conventions}

Throughout, we fix a separable Hilbert space $\mathcal{H}$. If we say $D$ is self-adjoint in $\mathcal{H}$, it is possibly unbounded and self-adjoint with a domain dense in $\mathcal{H}$. We let $\mathcal{B}(\mathcal{H})$ be the $\mathrm{C}^{*}$-algebra of bounded operators on $\mathcal{H}$. For $M \subseteq \mathcal{B}(\mathcal{H}), M_{\text {sa }}$ denotes the self-adjoint elements in $M$. We denote by $\mathcal{S}^{s} \subseteq \mathcal{B}(\mathcal{H})$ the Schatten class of $s$-summable operators, for $s \in \mathbb{N}=\{1,2, \ldots\}$. We write $\mathbb{N}_{0}=$ $\{0,1,2, \ldots\}$. 
Universal forms When considering a spectral triple $(\mathcal{A}, \mathcal{H}, D)$, we write $\Omega^{\bullet}(\mathcal{A})=\oplus_{n \in \mathbb{N}_{0}} \Omega^{n}(\mathcal{A})$ for the universal differential graded algebra over $\mathcal{A}=: \Omega^{0}(\mathcal{A})$, endowed with grading $d$. We write $\Omega_{D}^{1}(\mathcal{A}):=\pi_{D}\left(\Omega^{1}(\mathcal{A})\right)$ where $\pi_{D}: \Omega^{1}(\mathcal{A}) \rightarrow \mathcal{B}(\mathcal{H})$ is defined by (1) and (2). Whenever $A \in \Omega^{1}(\mathcal{A})$, we write $F:=d A+A^{2} \in \Omega^{2}(\mathcal{A})$ for the curvature of $A$.

Functions We write $C^{n}$ for the space of $n$ times continuously differentiable functions, $C:=C^{0}$, $C_{0}$ for the space of continuous functions vanishing at infinity, $C_{c}^{n}$ for the space of compactly supported functions in $C^{n}, \mathcal{D}:=C_{c}^{\infty}$ for the space of smooth compactly supported functions, $\mathcal{S}$ for the Schwartz space, and $L^{p}$ for the Lebesgue spaces $(p \in[1, \infty])$. All these functions are defined on $\mathbb{R}$ and have values in $\mathbb{C}$. We define $u(x):=x-i$, and write $u^{-1}$ for the multiplicative inverse of $u \in C$.

Fourier transforms We define the Fourier transform of an integrable function $f$ on $\mathbb{R}$ by $\hat{f}(x):=$ $\int_{\mathbb{R}} \frac{d y}{2 \pi} f(y) e^{-i y x}$. For a general (not necessarily tempered) distribution $f \in \mathcal{D}^{\prime}$ we can still define the Fourier transform as a distribution $\hat{f}: \hat{\mathcal{D}} \rightarrow \mathbb{C}$ by $\langle\hat{f} \mid \varphi\rangle:=\langle f \mid \hat{\varphi}\rangle$ for all Schwartz functions $\varphi$ with $\hat{\varphi} \in \mathcal{D}$. The restriction ${ }^{\wedge}: \mathcal{S}^{\prime} \rightarrow \mathcal{S}^{\prime}$ is bijective with inverse denoted by ${ }^{\vee}: \mathcal{S}^{\prime} \rightarrow \mathcal{S}^{\prime}$.

This more general definition of the Fourier transform will be applied in the following way. For an arbitrary continuous function $f$ (which is in $\mathcal{D}^{\prime}$ but not a priori in $\mathcal{S}^{\prime}$ ) we will often assume that $\hat{f} \in L^{1}$. Because then $\hat{f} \in \mathcal{S}^{\prime}$ and $\check{\hat{f}} \in C_{0}$ by the Riemann-Lebesgue lemma, we find that $\hat{\hat{f}}=\hat{f}$, which implies $\langle f \mid \varphi\rangle=\langle\check{f} \mid \varphi\rangle$ for all $\varphi \in \mathcal{D}$. Therefore $f=\check{f} \in C_{0}$ and $f(x)=\int \hat{f}(y) e^{i x y} d y$.

\section{Preliminaries on multiple operator integrals}

In this section, we introduce the subject of multiple operator integration in a sufficiently light way to fit our purposes. For a more extensive discussion, we refer to [1, 20, 23].

For every $n \in \mathbb{N}_{0}$ and $f \in C^{n}$, define the divided difference $f^{[n]}$ recursively as follows:

$$
\begin{aligned}
f^{[0]}\left(x_{0}\right) & :=f\left(x_{0}\right) \\
f^{[n+1]}\left(x_{0}, \ldots, x_{n+1}\right) & := \begin{cases}\frac{f^{[n]}\left(x_{0}, \ldots, x_{n}\right)-f^{[n]}\left(x_{0}, \ldots, x_{n-1}, x_{n+1}\right)}{x_{n}-x_{n+1}} & \text { if } x_{n} \neq x_{n+1} \\
\frac{\partial}{\partial x_{n}} f^{[n]}\left(x_{0}, \ldots, x_{n}\right) & \text { if } x_{n}=x_{n+1} .\end{cases}
\end{aligned}
$$

Let $\sigma$ denote the standard measure on the $n$-simplex,

$$
\Delta_{n}:=\left\{\left(s_{0}, \ldots, s_{n}\right) \in \mathbb{R}_{\geq 0}^{n+1}: \sum_{j=0}^{n} s_{j}=1\right\} .
$$

In order to define a multilinear operator integral, we need the following lemma.

Lemma 1. Whenever $f \in C^{n}$ is such that $\widehat{f^{(n)}} \in L^{1}$, we can write $f^{[n]}$ as

$$
f^{[n]}\left(x_{0}, \ldots, x_{n}\right)=\int_{\Delta_{n}} \int_{\mathbb{R}} e^{i t s_{0} x_{0}} \cdots e^{i t s_{n} x_{n}} \widehat{f^{(n)}}(t) d t d \sigma\left(s_{0}, \ldots, s_{n}\right) .
$$

Proof. One simply combines the proofs of [20, Lemma 5.1] and [20, Lemma 5.2]. 
It is easily seen that $\left(\Sigma, \sigma_{f}\right):=\left(\Delta_{n} \times \mathbb{R}, \sigma \times \widehat{f^{(n)}}\right)$ is a finite measure space with total variation equal to $\frac{1}{n !}\left\|\widehat{f^{(n)}}\right\|_{1}$.

Definition 2. Let $D_{0} \ldots, D_{n}$ be self-adjoint in $\mathcal{H}$ and let $f \in C^{n}$ such that $\widehat{f^{(n)}} \in L^{1}$. The multiple operator integral $T_{f^{[n]}}^{D_{0}, \ldots, D_{n}}: \mathcal{B}(\mathcal{H})^{\times n} \rightarrow \mathcal{B}(\mathcal{H})$ is defined on $V_{1}, \ldots, V_{n} \in \mathcal{B}(\mathcal{H})$ by

$$
T_{f^{[n]}}^{D_{0}, \ldots, D_{n}}\left(V_{1}, \ldots, V_{n}\right) y:=\int_{\Delta_{n}} \int_{\mathbb{R}} e^{i t s_{0} D_{0}} V_{1} e^{i t s_{1} D_{1}} \cdots V_{n} e^{i t s_{n} D_{n}} y \widehat{f^{(n)}}(t) d t d \sigma\left(s_{0}, \ldots, s_{n}\right),
$$

for $y \in \mathcal{H}$. In the case that $D_{j}=D$ for all $j$, we also write $T_{f^{[n]}}^{D}:=T_{f^{[n]}}^{D, \ldots, D}$.

This is a special case of the operator integral $T_{\phi}$ in [1, Definition 4.1] for the function $\phi=f^{[n]}$. We use the properties of $T_{\phi}$ proven there, e.g. we use [1, Lemma 4.3], to conclude that (5) does not depend on the representation of $f^{[n]}$ as given by Lemma 1. We also note that (5) in particular implies $T_{f^{[0]}}^{D}=f(D)$.

An important property of the multiple operator integral $T_{f^{[n]}}^{D}$ is that it allows us to compute a higher-order derivative of a trace functional like the spectral action:

$$
\left.\frac{1}{n !} \frac{d^{n}}{d t^{n}} \operatorname{Tr}(f(D+t V))\right|_{t=0}=\operatorname{Tr}\left(T_{f^{[n]}}^{D}(V, \ldots, V)\right),
$$

see for instance [1, Theorem 5.7]. Under other assumptions, this formula is proven in [23, Theorem 5.3.5], [17, Theorem 3.12] and [20, equation (5.30)]. In other words, multiple operator integrals are multilinear extensions of higher-order derivatives of the spectral action, which is why they are of interest to us. We write the Taylor series expansion of the spectral action as

$$
\operatorname{Tr}(f(D+V)) \sim \sum_{n=0}^{\infty} \operatorname{Tr}\left(T_{f^{[n]}}^{D}(V, \ldots, V)\right) .
$$

The remainder of this Taylor series can be written in terms of a single multiple operator integral:

Proposition 3. For any $K \in \mathbb{N}_{0}$, let $f \in C^{K+1}$ be such that $\widehat{f^{(n)}} \in L^{1}$ for $n \in\{0, \ldots, K+1\}$. Then

$$
f(D+V)-f(D)-\left.\sum_{n=1}^{K} \frac{1}{n !} \frac{d^{n}}{d t^{n}} f(D+t V)\right|_{t=0}=T_{f^{[K+1]}}^{D+V, D, \ldots, D}(V, \ldots, V) .
$$

Proof. The proof of [17, Theorem 3.13] exactly goes through for the stated class of functions.

The following lemma is an adaptation of [1, Lemma 4.6]. We give its proof for convenience of the reader.

Lemma 4. Fix $n \in \mathbb{N}_{0}$ and $f \in C^{n}$ such that $\widehat{f^{(n)}} \in L^{1}$, and let $D_{0}, \ldots, D_{n}$ be self-adjoint in $\mathcal{H}$. Suppose $\alpha, \alpha_{j} \in[1, \infty]$ with $\frac{1}{\alpha}=\frac{1}{\alpha_{1}}+\ldots+\frac{1}{\alpha_{n}}$, and let $V_{j} \in \mathcal{S}^{\alpha_{j}}$, where we set $\mathcal{S}^{\infty}=B(\mathcal{H})$. We then have

$$
\left\|T_{f^{[n]}}^{D_{0, \ldots, D_{n}}}\left(V_{1}, \ldots, V_{n}\right)\right\|_{\alpha} \leq \frac{1}{n !}\left\|\widehat{f^{(n)}}\right\|_{1}\left\|V_{1}\right\|_{\alpha_{1}} \cdots\left\|V_{n}\right\|_{\alpha_{n}} .
$$


Proof. Assume that $\alpha<\infty$. Define

$$
A_{s, t}:=e^{i t s_{0} D_{0}} V_{1} e^{i t s_{1} D_{1}} \cdots V_{n} e^{i t s_{n} D_{n}},
$$

for all $(s, t) \in \Delta_{n} \times \mathbb{R}=\Sigma$. Using Hölder's inequality we find that $A_{s, t} \in \mathcal{S}^{\alpha}$ and

$$
\left\|A_{s, t}\right\|_{\alpha} \leq\left\|V_{1}\right\|_{\alpha_{1}} \cdots\left\|V_{n}\right\|_{\alpha_{n}} .
$$

Let $B \in \mathcal{S}^{\alpha^{\prime}}$ be arbitrary, for $\alpha^{\prime}$ the Hölder conjugate exponent of $\alpha$. Define

$$
g(s, t):=A_{s, t} B,
$$

and notice that $g: \Sigma \rightarrow \mathcal{S}^{1}$ is uniformly $\mathcal{S}^{1}$-bounded. For all $j \leq n$, the map $(s, t) \mapsto e^{i t s_{j} D_{j}}$ is strongly continuous, and therefore $g: \Sigma \rightarrow \mathcal{S}^{1}$ is so*-continuous. By [1, Lemma 3.10], we obtain

$$
\begin{aligned}
\left|\operatorname{Tr}\left(T_{f^{[n]}}^{D_{0}, \ldots, D_{n}}\left(V_{1}, \ldots, V_{n}\right) B\right)\right| & =\left|\int_{\Sigma} \operatorname{Tr}\left(A_{s, t} B\right) d \sigma_{f}(s, t)\right| \\
& \leq \int_{\Sigma} \operatorname{Tr}\left|A_{s, t} B\right| d\left|\sigma_{f}\right|(s, t) \\
& \leq\left\|V_{1}\right\|_{\alpha_{1}} \cdots\left\|V_{n}\right\|_{\alpha_{n}}\|B\|_{\alpha^{\prime}} \frac{1}{n !}\left\|\widehat{f^{(n)}}\right\|_{\infty} .
\end{aligned}
$$

When $\alpha=1$, simply taking a unitary $B$ such that $T_{f^{[n]}}^{D_{0,}, D_{n}}\left(V_{1}, \ldots, V_{n}\right) B=\left|T_{f^{[n]}}^{D_{0}, \ldots, D_{n}}\left(V_{1}, \ldots, V_{n}\right)\right|$ yields the result. If $\alpha \in(1, \infty)$, then an application of $\left(\mathcal{S}^{\alpha^{\prime}}\right)^{*}=\mathcal{S}^{\alpha}$ gives the result. If $\alpha=\infty$, one obtains the lemma directly by the triangle inequality $\left\|\int A_{s, t}\right\| \leq \int\left\|A_{s, t}\right\|$.

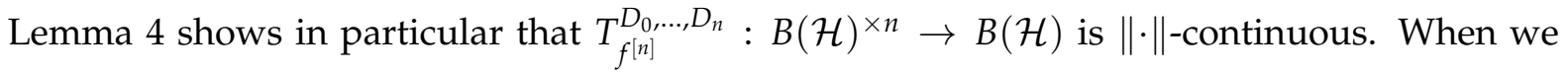
replace $(B(\mathcal{H}),\|\cdot\|)$ by $\left(B(\mathcal{H})_{1}\right.$, s.o.t.), this is known to still hold true (see [1, Proposition 4.9]). These results can be unified and generalized by writing $\mathcal{L}^{\alpha}:=\left(\mathcal{S}^{\alpha},\|\cdot\|_{\alpha}\right)$ for $\alpha \in[1, \infty)$ and $\mathcal{L}^{\infty}:=$ $\left(B(\mathcal{H})_{1}\right.$, s.o.t. $)$. We then have the following.

Lemma 5. Let $f \in C^{n}$ with $\widehat{f^{(n)}} \in L^{1}$ and let $\alpha, \alpha_{j} \in[1, \infty]$ with $\frac{1}{\alpha}=\frac{1}{\alpha_{1}}+\ldots+\frac{1}{\alpha_{n}}$. Assume that either $\alpha_{n}<\infty$ or $\alpha_{1}=\ldots=\alpha_{n}=\infty$. Then

$$
T_{f^{[n]}}^{D_{0}, \ldots, D_{n}}: \mathcal{L}^{\alpha_{1}} \times \cdots \times \mathcal{L}^{\alpha_{n}} \rightarrow \mathcal{L}^{\alpha}
$$

is continuous.

Proof. If $\alpha=\infty$, then all $\alpha_{j}=\infty$, and the result is proven in [1, Proposition 4.9]. If $\alpha<\infty$, we can use the same technique and notation as in the proof of Lemma 4 to find

$$
\operatorname{Tr}\left(T_{f^{[n]}}^{D_{0,}, \ldots, D_{n}}\left(V_{1}, \ldots, V_{n}\right) B\right)=\int_{\Sigma} \operatorname{Tr}\left(A_{s, t} B\right) d \sigma_{f}(s, t),
$$

for every $B \in \mathcal{S}^{\alpha^{\prime}}$. By Hölder's inequality and the continuity of the product $\mathcal{B}(\mathcal{H})_{1} \times \mathcal{L}^{p} \rightarrow \mathcal{L}^{p}$,

$$
\mathcal{L}^{p} \times \mathcal{L}^{q} \rightarrow \mathcal{L}^{r}, \quad(A, B) \mapsto A B
$$


is continuous for every $p, q, r \in[1, \infty]$ with $\frac{1}{p}+\frac{1}{q}=\frac{1}{r}$ and either $q<\infty$ or $p=q=\infty$. Therefore also the product $\mathcal{L}^{p} \times\left(\mathcal{L}^{q} \times \mathcal{L}^{s}\right) \rightarrow \mathcal{L}^{r}$ is continuous whenever $p, q, r, s \in[1, \infty]$ satisfy $\frac{1}{p}+\frac{1}{q}+\frac{1}{s}=$ $\frac{1}{r}$ and either $s<\infty$ or $p=q=s=\infty$. By repeatedly applying this continuity property, we find that the product

$$
\mathcal{L}^{\alpha_{1}} \times \cdots \times \mathcal{L}^{\alpha_{n}} \rightarrow \mathcal{L}^{\alpha}, \quad\left(V_{1}, \ldots, V_{n}\right) \mapsto V_{1} \cdots V_{n}
$$

is continuous under the standing assumptions on $\alpha, \alpha_{j}$. Hence, for every $(s, t) \in \Sigma$, the operator $A_{s, t} B \in \mathcal{L}^{1}$ depends continuously on $\left(V_{1}, \ldots, V_{n}\right) \in \mathcal{L}^{\alpha_{1}} \times \cdots \times \mathcal{L}^{\alpha_{n}}$. Since a convergent sequence in $\mathcal{L}^{p}$ is bounded w.r.t. $\|\cdot\|_{p}$ (where $\|\cdot\|_{\infty}=\|\cdot\|$ ) an application of the dominated convergence theorem shows that $(9)$ depends sequentially continuously on $\left(V_{1}, \ldots, V_{n}\right)$. By our specific choice of $\mathcal{L}^{\infty}$, every $\mathcal{L}^{p}$ is a metric space, hence sequential continuity implies continuity.

The relation $\frac{1}{\alpha}=\frac{1}{\alpha_{1}}+\ldots+\frac{1}{\alpha_{n}}$ is central to the above Lemma. When the resolvent of $D$ is sSchatten, however, for an explicit class $\mathcal{W}^{s, n}$ of functions $f$, defined in Section 3 , we can do away with this relation. We will prove this in $\$ 3.2$.

\section{Estimating the multiple operator integral in the $s$-summable case}

We specialize the class of functions $f$ that appear in Proposition 3 and consider for $s, n \in \mathbb{N}_{0}$ :

$$
\mathcal{W}^{s, n}:=\left\{f \in C^{n}: \widehat{\left(f u^{m}\right)^{(k)}} \in L^{1} \text { for all } m \leq s \text { and } k \leq n\right\},
$$

where $u(x):=x-i$. Examples of functions in $\mathcal{W}^{s, n}$ are $n+1$-differentiable functions such that $\left(f u^{s}\right)^{(k)} \in L^{2}$ for all $k \leq n+1$, such as Schwartz functions, or functions in $C_{c}^{n+1}$.

The main result of this section will be the following bound on the multiple operator integral.

Theorem 6. Let $D$ be self-adjoint in $\mathcal{H}$ such that $(D-i)^{-1} \in \mathcal{S}^{s}$ for $s \in \mathbb{N}$. For every $n \in \mathbb{N}_{0}$, every $f \in \mathcal{W}^{s, n}$ and every $V_{1}, \ldots, V_{n} \in B(\mathcal{H})$, the multiple operator integral $T_{f[n]}^{D}\left(V_{1}, \ldots, V_{n}\right)$ is trace-class and satisfies the bound

$$
\left\|T_{f^{[n]}}^{D}\left(V_{1}, \ldots, V_{n}\right)\right\|_{1} \leq c_{s, n}(f)\left\|V_{1}\right\| \cdots\left\|V_{n}\right\|\left\|(D-i)^{-1}\right\|_{s^{\prime}}^{s}
$$

where

$$
c_{s, n}(f):=\sum_{k=0}^{\min (s, n)}\left(\begin{array}{l}
s \\
k
\end{array}\right) \frac{\|\left(f \widehat{\left.\left.u^{s-k}\right)^{(n}-k\right)} \|_{1}\right.}{(n-k) !} .
$$

More generally, when $V \in \mathcal{B}(\mathcal{H})_{\mathrm{sa}}$,

$$
\left\|T_{f^{[n]}}^{D+V, D, \ldots, D}\left(V_{1}, \ldots, V_{n}\right)\right\|_{1} \leq c_{s, n}(f)\left\|V_{1}\right\| \cdots\left\|V_{n}\right\|(1+\|V\|)^{2 s}\left\|(D-i)^{-1}\right\|_{s}^{s} .
$$

This theorem allows us to freely work with the traces of multiple operator integrals up to order $n$. This is the sole analytical ingredient for a truncated version of our main result, namely Theorem 29. However, if we want the expansion (4) to converge, we will need to impose infinite 
differentiability of $f$, as well as a growth condition on the $L^{1}$-norms occurring in Theorem 6 , as $n$ goes to infinity. We therefore introduce the space

$$
\mathcal{E}^{s, \gamma}:=\left\{f \in C^{\infty}: \text { there exists } C_{f} \geq 1 \text { s.t. }\left\|\widehat{\left(f u^{m}\right)^{(n)}}\right\|_{1} \leq C_{f}^{n+1} n !^{\gamma} \text { for all } m \leq s \text { and } n \in \mathbb{N}_{0}\right\},
$$

for $\gamma \in(0,1]$. Our main result is that the expansion (4) holds for all functions $f \in \mathcal{E}^{s, \gamma}$, and certain perturbations $A$. If $\gamma=1$, the expansion converges absolutely whenever the perturbation $A$ is sufficiently small. If $\gamma<1$ the expansion converges absolutely for all perturbations. The following Lemma underlies both results.

Lemma 7. Let $s \in \mathbb{N}, D$ self-adjoint in $\mathcal{H}$ with $(D-i)^{-1} \in \mathcal{S}^{s}$, and $\gamma \in(0,1]$. For any $f \in \mathcal{E}^{s, \gamma}$ there exists a $C \geq 1$ such that for all $n \in \mathbb{N}_{0}, V_{1}, \ldots, V_{n} \in \mathcal{B}(\mathcal{H})$, and $V \in \mathcal{B}(\mathcal{H})_{\text {sa, }}$, we have

$$
\left\|T_{f^{[n]}}^{D+V, D, \ldots, D}\left(V_{1}, \ldots, V_{n}\right)\right\|_{1} \leq\left(C^{n+1} n !^{\gamma-1}\right)\left\|V_{1}\right\| \cdots\left\|V_{n}\right\|(1+\|V\|)^{2 s}\left\|(D-i)^{-1}\right\|_{s}^{s} .
$$

Proof. Apply the definition of $\mathcal{E}^{s, \gamma}$ to Theorem 6 , and absorb $2^{s}$ into the constant $C$.

This lemma will be used in Subsection 5.2 and Section 6 .

Examples of functions in $\mathcal{E}^{s, 1}$ are Schwartz functions with compactly supported Fourier transform. The following proposition gives more examples.

Proposition 8. Let $f \in C^{\infty}$ and $s, t \in \mathbb{N}_{0}$.

(i) If $f \in \mathcal{E}^{s, 1}$ and $g \in \mathcal{E}^{t, 1}$, then $f g \in \mathcal{E}^{s+t, 1}$.

(ii) If $\hat{f} \in L^{1}$ with $|\hat{f}(x)| \leq e^{-c|x|}$ a.e. for some $c>0$, then $f \in \mathcal{E}^{0,1}$.

(iii) If $\widehat{f u^{s}} \in L^{1}$ with $\left|\widehat{f u^{s}}(x)\right| \leq e^{-c|x|}$ a.e. for some $c>0$, then $f \in \mathcal{E}^{s, 1}$.

(iv) Rational functions in $\mathcal{O}\left(|x|^{-s-1}\right)$ are in $\mathcal{E}^{s, 1}$.

(v) The function $x \mapsto e^{-x^{2}}$ is in $\mathcal{E}^{s, 1 / 2}$ for any $s \in \mathbb{N}_{0}$.

Proof. $\quad$ (i) For $m \leq s$ and $p \leq t$, Young's inequality gives

$$
\begin{aligned}
\left\|\left(f g \widehat{u^{m+p}}\right)^{(n)}\right\|_{1} & \leq \sum_{k=0}^{n}\left(\begin{array}{l}
n \\
k
\end{array}\right)\left\|\left(\widehat{\left.f u^{m}\right)^{(k}}\right)\right\|_{1} \|\left(\widehat{\left.g u^{p}\right)^{(n-k}} \|_{1}\right. \\
& \leq(n+1) n !\left(C_{f} C_{g}\right)^{n+1} .
\end{aligned}
$$

Any polynomial in $n$ is $\mathcal{O}\left(C^{n}\right)$ for some $C \geq 1$.

(ii) As

$$
\frac{1}{n !}\left(\frac{1}{2} c\right)^{n}|x|^{n} \leq \sum_{m=0}^{\infty} \frac{1}{m !}\left(\frac{1}{2} c|x|\right)^{m}=e^{\frac{1}{2} c|x|}
$$

we find $\left\|\widehat{f^{(n)}}\right\|_{1}=\left\||x|^{n} \hat{f}\right\|_{1} \leq\left\|e^{\frac{1}{2} c|x|} \hat{f}\right\|_{1}\left(\frac{1}{2} c\right)^{-n} n !$, thereby obtaining $f \in \mathcal{E}^{0,1}$. 
(iii) Item (ii) gives that $f u^{s} \in \mathcal{E}^{0,1}$. It is easy to see that $u^{-s} \in \mathcal{E}^{s-1,1}$. Therefore (i) gives $f \in \mathcal{E}^{s-1,1}$, i.e., $\|\left(\widehat{\left.f u^{m}\right)^{(n)}} \|_{1} \leq C_{f}^{n+1} n\right.$ ! for $m \leq s-1$. Similar to (ii) we get $\left\|\widehat{\left(f u^{s}\right)^{(n)}}\right\|_{1} \leq C^{n+1} n$ ! for some $C \geq 1$.

(iv) Follows from (iii),

(v) Let $f(x)=e^{-x^{2}}$ and $m \in \mathbb{N}_{0}$. The Fourier transform of $f u^{m}$ is a polynomial times a Gaussian, say $\left(f u^{m}\right)^{\wedge}(x)=p(x) e^{-x^{2} / c^{2}}$. Therefore,

$$
\|\left(\widehat{\left.f u^{m}\right)^{(n)}}\left\|_{1}=\right\||x|^{n} p(x) e^{-x^{2} / c^{2}} \|_{1} .\right.
$$

Furthermore, $|x|^{n}=c^{n} \sqrt{\left(x^{2} / c^{2}\right)^{n}} \leq c^{n} \sqrt{n ! e^{x^{2} / c^{2}}}=\sqrt{n !} c^{n} e^{\frac{x^{2}}{2 c^{2}}}$, so

$$
\left\|\widehat{\left(f u^{m}\right)^{(n)}}\right\|_{1} \leq \sqrt{n !} c^{n}\left\|p(x) e^{-\frac{x^{2}}{2 c^{2}}}\right\|_{1}
$$

Therefore, $f \in \mathcal{E}^{s, 1 / 2}$ for any $s \in \mathbb{N}_{0}$.

\subsection{Bound on the multiple operator integral}

We will now give a proof of Theorem 6 , which uses the summability of $D$ to obtain a trace class estimate on the multiple operator integral $T_{f^{[n]}}^{D}$. For summability $s=2$, a similar estimate was found by Anna Skripka in [22, Lemma 3.6]. This was later generalized to the case of so-called relative Schatten perturbations in [17, Theorem 3.10], but only holds for $k$-multilinear operator integrals of order $k \geq s$.

The core idea of our proof is the same as of [22, Lemma 3.6] and [17, Theorem 3.10], namely to expand $T_{f[n]}\left(V_{1}, \ldots, V_{n}\right)$ as a sum of operator integrals, which should then be bounded using the triangle inequality and (a noncommutative) Hölder's inequality. For brevity, we sometimes write $V_{k}^{\{j\}}:=V_{k}\left(D_{k}-i\right)^{-j}$, and $T_{\phi}^{D_{0}, \ldots, D_{k}}\left(V_{1}, \ldots, V_{k}\right)^{\{j\}}:=T_{\phi}^{D_{0}, \ldots, D_{k}}\left(V_{1}, \ldots, V_{k}\right)\left(D_{k}-i\right)^{-j}$.

Lemma 9. For $s, n \in \mathbb{N}_{0}, f \in \mathcal{W}^{s, n}, V_{1}, \ldots, V_{n} \in \mathcal{B}(\mathcal{H})$, and $D_{0}, \ldots, D_{n}$ self-adjoint in $\mathcal{H}$, we have

$$
T_{f^{[n]}}^{D_{0}, \ldots, D_{n}}\left(V_{1}, \ldots, V_{n}\right)=\sum_{k=0}^{\min (s, n)}(-1)^{k} \sum_{\substack{j_{0} \geq 0, j_{1}, \ldots, j_{k} \geq 1, j_{0}+\ldots+j_{k}=s}} T_{\left(f u^{s-k}\right)^{[n-k]}}^{D_{0, \ldots, D^{n-k}}}\left(V_{1}, \ldots, V_{n-k}\right)^{\left\{j_{0}\right\}} V_{n-k+1}^{\left\{j_{1}\right\}} \cdots V_{n}^{\left\{j_{k}\right\}} .
$$

Proof. We prove the lemma by induction on $s$. If $s=0$, the statement follows easily. For the induction step, we note that the proof of [17, Theorem 3.10(i)] implies that, for all $f \in \mathcal{W}^{s, n}$, we have

$$
T_{f^{[n]}}^{D_{0}, \ldots, D_{n}}\left(V_{1}, \ldots, V_{n}\right)=T_{(f u)^{[n]}}^{D_{0}, \ldots, D_{n}}\left(V_{1}, \ldots, V_{n}\right)\left(D_{n}-i\right)^{-1}-T_{f^{[n-1]}}^{D_{0}, \ldots, D_{n-1}}\left(V_{1}, \ldots, V_{n-1}\right) V_{n}\left(D_{n}-i\right)^{-1} .
$$


Suppose the claim of the lemma holds for a certain $s \in \mathbb{N}_{0}$. Then

$$
\begin{aligned}
T_{f}^{[n]} & D_{0, \ldots, D_{n}}\left(V_{1}, \ldots, V_{n}\right) \\
= & \sum_{k=0}^{\min (s, n)} \sum_{\substack{j_{0} \geq 0, j_{1}, \ldots, j_{k} \geq 1 \\
j_{0}+\ldots+j_{k}=s}}(-1)^{k} T_{\left(f u^{s-k+1}\right)^{[n-k]}}^{D_{0}, \ldots, D_{n-k}}\left(V_{1}, \ldots, V_{n-k}\right)^{\left\{j_{0}+1\right\}} V_{n-k+1}^{\left\{j_{1}\right\}} \cdots V_{n}^{\left\{j_{k}\right\}} \\
& +\sum_{k=0}^{\min (s, n-1)} \sum_{\substack{j_{0} \geq 0, j_{1}, \ldots, j_{k} \geq 1 \\
j_{0}+\ldots+j_{k}=s}}(-1)^{k+1} T_{\left(f u^{-k-k}\right)^{[n-k-1]}}^{D_{0}, \ldots, D_{n-k-1}}\left(V_{1}, \ldots, V_{n-k-1}\right) V_{n-k}^{\left\{j_{0}+1\right\}} V_{n-k+1}^{\left\{j_{1}\right\}} \cdots V_{n}^{\left\{j_{k}\right\}} \\
& =\sum_{k=0}^{\min (s, n)} \sum_{\substack{j_{0} \geq 1, j_{1}, \ldots, j_{k} \geq 1 \\
j_{0}+\ldots+j_{k}=s+1}}(-1)^{k} T_{\left(f u^{s+1-k}\right)^{[n-k]}}^{D_{0}, \ldots, D_{n-k}}\left(V_{1}, \ldots, V_{n-k}\right)^{\left\{j_{0}\right\}} V_{n_{k}+1}^{\left\{j_{1}\right\}} \cdots V_{n}^{\left\{j_{k}\right\}} \\
& +\sum_{k=1}^{\min (s+1, n)} \sum_{\substack{j_{0}=0, j_{1}, \ldots, j_{k} \geq 1 \\
j_{0}+\ldots+j_{k}=s+1}}(-1)^{k} T_{\left(f u^{s+1-k}\right)^{[n-k]}}^{D_{0}, \ldots, D_{n-k}}\left(V_{1}, \ldots, V_{n-k}\right)^{\left\{j_{0}\right\}} V_{n-k+1}^{\left\{j_{1}\right\}} \cdots V_{n}^{\left\{j_{k}\right\}} .
\end{aligned}
$$

In the first term we can freely replace the sum from $k=0$ to $\min (s, n)$ by a sum from $k=0$ to $\min (s+1, n)$, because the appearing sum over $j_{0}, \ldots, j_{k}$ is trivial for $k=s+1$. Similarly, in the second term, we can freely let $k$ run from 0 to $\min (s+1, n)$. Combining the two terms gives the claim of the lemma for $s+1$, which completes the induction step.

We prove the main result of this section.

Proof of Theorem 6 . We apply Lemma 9 , and find

$$
\left\|T_{f^{[n]}}^{D}\left(V_{1}, \ldots, V_{n}\right)\right\|_{1} \leq \sum_{k=0}^{\min (s, n)} \sum_{\substack{j_{0} \geq 0, j_{1}, \ldots, j_{k} \geq 1 \\ j_{0}+\ldots+j_{k}=s}}\left\|T_{\left(f u^{s-k}\right)^{[n-k]}}^{D}\left(V_{1}, \ldots, V_{n-k}\right)\left\{j_{0}\right\}\right\|\left\|_{\frac{s}{j_{0}}}\right\| V_{n-k+1}^{\left\{j_{1}\right\}}\left\|_{\frac{s}{j_{1}}} \ldots\right\| V_{n}^{\left\{j_{k}\right\}} \|_{\frac{s}{j_{k}}} .
$$

Apply Lemma 4 , to find

$$
\left\|T_{f^{[n]}}^{D}\left(V_{1}, \ldots, V_{n}\right)\right\|_{1} \leq \sum_{k=0}^{\min (s, n)} \sum_{\substack{j_{0} \geq 0, j_{1}, \ldots, j_{k} \geq 1 \\ j_{0}+\ldots+j_{k}=s}} \frac{1}{(n-k) !}\left\|\left(f \widehat{\left.u^{s-k}\right)^{(n}}-k\right)\right\|_{1}\left\|V_{1}\right\| \cdots\left\|V_{n}\right\|\left\|(D-i)^{-1}\right\|_{s}^{s} .
$$

A bit of combinatorics shows that the sum over $j_{0}, \ldots, j_{k}$ adds a factor $\left(\begin{array}{l}s \\ k\end{array}\right)$, which implies the first statement of the theorem. The second statement follows similarly, with the added remark that

$$
\left\|(D+V-i)^{-1}\right\|_{s}^{s} \leq(1+\|V\|)^{2 s}\left\|(D-i)^{-1}\right\|_{s}^{s} .
$$

See, e.g., [14, Appendix B, Lemma 6]. 


\subsection{Continuity of the multiple operator integral}

Theorem 10. Let $s \in \mathbb{N}, D$ self-adjoint in $\mathcal{H}$ with $(D-i)^{-1} \in \mathcal{S}^{s}, n \in \mathbb{N}_{0}$, and $f \in \mathcal{W}^{s, n}$. The map

$$
T_{f^{[n]}}^{D}: \mathcal{L}^{\infty} \times \cdots \times \mathcal{L}^{\infty} \rightarrow \mathcal{L}^{1}
$$

is continuous. (Recall that $\mathcal{L}^{\infty}=B(\mathcal{H})_{1}$, endowed with the strong operator topology.)

Proof. Suppose that $V_{1}^{m} \rightarrow V_{1}, \ldots, V_{n}^{m} \rightarrow V_{n}$ in $\mathcal{L}^{\infty}$. By continuity of (10), we obtain that

$$
\left(V_{n-k+l}^{m}\right)^{\left\{j_{l}\right\}} \rightarrow V_{n-k+l}^{\left\{j_{l}\right\}} \text { in } \mathcal{L}^{s / j_{l}} .
$$

We invoke Lemma 5 to find that

$$
T_{\left(f u^{s-k}\right)^{[n-k]}}^{D}\left(V_{1}^{m}, \ldots, V_{n-k}^{m}\right)^{\left\{j_{0}\right\}} \rightarrow T_{\left(f u^{s-k}\right)^{[n-k]}}^{D}\left(V_{1}, \ldots, V_{n-k}\right)^{\left\{j_{0}\right\}} \quad \text { in } \mathcal{L}^{s / j_{0}} .
$$

By Lemma 9 and the continuity of the product (11) (for which we remark that the assumptions on $\alpha_{j}, \alpha$ are indeed satisfied for all terms obtained from Lemma 9 ) we find that

$$
T_{f^{[n]}}^{D}\left(V_{1}^{m}, \ldots, V_{n}^{m}\right) \rightarrow T_{f^{[n]}}^{D}\left(V_{1}, \ldots, V_{n}\right) \quad \text { in } \mathcal{L}^{1},
$$

so we are done.

To emphasize the strength of this result, we compare it to Lemma 5 which is (at least in the separate cases $\alpha_{1}, \ldots, \alpha_{n}<\infty$ and $\alpha=\infty$ ) known in the literature. Using the continuity of the inclusion $\mathcal{L}^{\alpha} \hookrightarrow \mathcal{L}^{\infty}$ we obtain the following clear improvement.

Corollary 11. Let $s \in \mathbb{N}, D$ self-adjoint in $\mathcal{H}$ with $(D-i)^{-1} \in \mathcal{S}^{s}, n \in \mathbb{N}_{0}$, and $f \in \mathcal{W}^{s, n}$. For any $\alpha_{1}, \ldots, \alpha_{n} \in[1, \infty]$ and any $\alpha \in[1, \infty]$ (no relation between $\alpha$ and the $\alpha_{j}$ 's assumed) the map

$$
T_{f^{[n]}}^{D}: \mathcal{L}^{\alpha_{1}} \times \cdots \times \mathcal{L}^{\alpha_{n}} \rightarrow \mathcal{L}^{\alpha}
$$

is continuous.

\section{Cyclic cocycles and universal forms underlying the spectral action}

Mainly to fix our conventions, we start with the definition of a finitely summable spectral triple, which is the situation in which our main result is stated.

Definition 12. Let $s \in \mathbb{N}$. An s-summable spectral triple $(\mathcal{A}, \mathcal{H}, D)$ consists of a separable Hilbert space $\mathcal{H}$, a self-adjoint operator $D$ in $\mathcal{H}$ and a unital *algebra $\mathcal{A} \subseteq B(\mathcal{H})$, such that, for all $a \in \mathcal{A}$, $a \operatorname{dom} D \subseteq \operatorname{dom} D$ and $[D, a]$ extends to a bounded operator, and $(D-i)^{-1} \in \mathcal{S}^{s}$.

Throughout this section, we let $(\mathcal{A}, \mathcal{H}, D)$ be an $s$-summable spectral triple for $s \in \mathbb{N}$, and we let $f \in \mathcal{W}^{s, n}$ for $n \in \mathbb{N}_{0}$, unless stated otherwise.

Definition 13. Define a multilinear function $\langle\cdot\rangle: B(\mathcal{H})^{\times n} \rightarrow \mathbb{C}$ by

$$
\left\langle V_{1}, \ldots, V_{n}\right\rangle:=\sum_{j=1}^{n} \operatorname{Tr}\left(T_{f^{[n]}}^{D}\left(V_{j}, \ldots, V_{n}, V_{1}, \ldots, V_{j-1}\right)\right) .
$$


For our algebraic results (which make up most of Section 4 and \$5.1) we only need two simple properties of the bracket $\langle\cdot\rangle$, stated in the following lemma. After proving this lemma, all analytical subtleties (related to the unboundedness of $D$ ) are taken care of, and we can focus on the algebra that ensues from these simple rules.

Lemma 14. For $V_{1}, \ldots, V_{n} \in \mathcal{B}(\mathcal{H})$ and $a \in \mathcal{A}$ we have

(I) $\left\langle V_{1}, \ldots, V_{n}\right\rangle=\left\langle V_{n}, V_{1}, \ldots, V_{n-1}\right\rangle$,

(II) $\left\langle V_{1}, \ldots, a V_{j}, \ldots, V_{n}\right\rangle-\left\langle V_{1}, \ldots, V_{j-1} a, \ldots, V_{n}\right\rangle=\left\langle V_{1}, \ldots, V_{j-1},[D, a], V_{j}, \ldots, V_{n}\right\rangle$,

where it is understood that for the edge case $j=1$ we need to substitute $n$ for $j-1$ on the left-hand side, and $f \in \mathcal{W}^{s, n+1}$ is assumed to define the right-hand side.

Proof. Property (I) follows immediately from Definition 13. By writing out the definitions for rank-1 operators $V_{1}, \ldots, V_{n}$, we have,

$$
\begin{aligned}
& T_{f^{[n]}}^{D}\left(V_{1}, \ldots, V_{j}, a V_{j+1}, \ldots, V_{n}\right)-T_{f^{[n]}}^{D}\left(V_{1}, \ldots, V_{j} a, V_{j+1}, \ldots, V_{n}\right) \\
& \quad=T_{f^{[n+1]}}^{D}\left(V_{1}, \ldots, V_{j},[D, a], V_{j+1}, \ldots, V_{n}\right)
\end{aligned}
$$

and the two edge cases,

$$
\begin{aligned}
& T_{f^{[n]}}^{D}\left(a V_{1}, \ldots, V_{n}\right)-a T_{f^{[n]}}^{D}\left(V_{1}, \ldots, V_{n}\right)=T_{f^{[n+1]}}^{D}\left([D, a], V_{1}, \ldots, V_{n}\right), \\
& T_{f^{[n]}}^{D}\left(V_{1}, \ldots, V_{n}\right) a-T_{f^{[n]}}^{D}\left(V_{1}, \ldots, V_{n} a\right)=T_{f^{[n+1]}}^{D}\left(V_{1}, \ldots, V_{n},[D, a]\right) .
\end{aligned}
$$

By Theorem 10 , and the fact that the finite-rank operators lie strongly dense in $B(\mathcal{H})$, we find that formulas (13), (14) and (15) hold for all $V_{1}, \ldots, V_{n} \in B(\mathcal{H})$. Hence,

$$
\begin{aligned}
\left\langle a V_{1},\right. & \left.V_{2}, \ldots, V_{n}\right\rangle-\left\langle V_{1}, V_{2}, \ldots, V_{n} a\right\rangle \\
= & \sum_{j=2}^{n} \operatorname{Tr}\left(T_{f^{[n]}}^{D}\left(V_{j}, \ldots, V_{n},[D, a], V_{1}, \ldots, V_{j-1}\right)\right) \\
& +\operatorname{Tr}\left(T_{f^{[n]}}^{D}\left(a V_{1}, \ldots, V_{n}\right)\right)-\operatorname{Tr}\left(T_{f^{[n]}}^{D}\left(V_{1}, \ldots, V_{n} a\right)\right) \\
= & \sum_{j=2}^{n} \operatorname{Tr}\left(T_{f^{[n+1]}}^{D}\left(V_{j}, \ldots, V_{n},[D, a], V_{1}, \ldots, V_{j-1}\right)\right) \\
& +\operatorname{Tr}\left(T_{f^{[n+1]}}^{D}\left([D, a], V_{1}, \ldots, V_{n}\right)\right)+\operatorname{Tr}\left(a T_{f^{[n]}}^{D}\left(V_{1}, \ldots, V_{n}\right)\right)-\operatorname{Tr}\left(T_{f^{[n]}}^{D}\left(V_{1}, \ldots, V_{n} a\right)\right) \\
= & \sum_{j=2}^{n} \operatorname{Tr}\left(T_{f^{[n+1]}}^{D}\left(V_{j}, \ldots, V_{n},[D, a], V_{1}, \ldots, V_{j-1}\right)\right) \\
& +\operatorname{Tr}\left(T_{f^{[n+1]}}^{D}\left([D, a], V_{1}, \ldots, V_{n}\right)\right)+\operatorname{Tr}\left(T_{f^{[n+1]}}^{D}\left(V_{1}, \ldots, V_{n},[D, a]\right)\right) \\
= & \left\langle[D, a], V_{1}, \ldots, V_{n}\right\rangle,
\end{aligned}
$$

and therefore (II) follows by applying (I) 
Remark 15. Under additional assumptions - for instance when $V_{1}, \ldots, V_{n} \in \mathcal{S}^{1}$ and $f \in \mathcal{W}^{s, n}$ is such that $f^{\prime}$ is compactly supported and analytic in a region of $\mathbb{C}$ containing a rectifiable curve $\gamma$ which surrounds the support of $f$ in $\mathbb{R}$ - we have

$$
\left\langle V_{1}, \ldots, V_{n}\right\rangle=\frac{1}{2 \pi i} \operatorname{Tr} \oint_{\gamma} f^{\prime}(z) \prod_{j=1}^{n} V_{j}(z-D)^{-1}
$$

This occurs in [24, Corl. 20] in the case where $V_{1}=V_{2}=\cdots=V_{n}$. It would be interesting to confront these resolvent formulas with the ones appearing in the work of Paycha [19].

\subsection{Hochschild and cyclic cocycles}

When the above brackets $\langle\cdot\rangle$ are evaluated at one-forms $a[D, b]$ associated to a spectral triple, the relations found in Lemma 14 can be translated nicely in terms of the coboundary operators appearing in cyclic cohomology. This is very similar to the structure appearing in the context of index theory, see for instance [15, 16].

Let us start by recalling the definition of the boundary operators $b$ and $B$ from [7].

Definition 16. If $\mathcal{A}$ is an algebra, and $n \in \mathbb{N}_{0}$, we define the space of Hochschild $n$-cochains, denoted by $\mathcal{C}^{n}(\mathcal{A})$, as the space of $(n+1)$-linear functionals $\phi$ on $\mathcal{A}$ with the property that if $a_{j}=1$ for some $j \geq 1$, then $\phi\left(a_{0}, \ldots, a_{n}\right)=0$. Define operators $b: \mathcal{C}^{n}(\mathcal{A}) \rightarrow \mathcal{C}^{n+1}(\mathcal{A})$ and $B: \mathcal{C}^{n+1}(\mathcal{A}) \rightarrow \mathcal{C}^{n}(\mathcal{A})$ by

$$
\begin{aligned}
b \phi\left(a_{0}, a_{1}, \ldots, a_{n+1}\right):= & \sum_{j=0}^{n}(-1)^{j} \phi\left(a_{0}, \ldots, a_{j} a_{j+1}, \ldots, a_{n+1}\right) \\
& +(-1)^{n+1} \phi\left(a_{n+1} a_{0}, a_{1}, \ldots, a_{n}\right), \\
B \phi\left(a_{0}, a_{1}, \ldots, a_{n}\right):= & \sum_{j=0}^{n}(-1)^{n j} \phi\left(1, a_{j}, a_{j+1}, \ldots, a_{j-1}\right) .
\end{aligned}
$$

Note that $B=A B_{0}$ in terms of the operator $A$ of cyclic anti-symmetrization and the operator defined by $B_{0} \phi\left(a_{0}, a_{1}, \ldots, a_{n}\right)=\phi\left(1, a_{0}, a_{1}, \ldots, a_{n}\right)$.

One may check that the pair $(b, B)$ defines a double complex, i.e. $b^{2}=0, B^{2}=0$ and $b B+B b=$ 0 . Hochschild cohomology then arises as the cohomology of the complex $\left(\mathcal{C}^{n}(\mathcal{A}), b\right)$, while the for us relevant periodic cyclic cohomology is defined as the cohomology of the totalization of the $(b, B)$-complex. That is to say,

$$
\mathcal{C}^{\mathrm{ev}}(\mathcal{A})=\bigoplus_{k} \mathcal{C}^{2 k}(\mathcal{A}) ; \quad \mathcal{C}^{\mathrm{odd}}(\mathcal{A})=\bigoplus_{k} \mathcal{C}^{2 k+1}(\mathcal{A}),
$$

form a complex with differential $b+B$ and the cohomology of this complex is called periodic cyclic cohomology. We will also refer to a periodic cyclic cocycle as a $(b, B)$-cocycle. Explicitly, an odd $(b, B)$-cocycle is thus given by a sequence

$$
\left(\phi_{1}, \phi_{3}, \phi_{5}, \ldots\right)
$$

where $\phi_{2 k+1} \in \mathcal{C}^{2 k+1}(\mathcal{A})$ and

$$
b \phi_{2 k+1}+B \phi_{2 k+3}=0,
$$

for all $k \geq 0$, and also $B \phi_{1}=0$. An analogous statement holds for even $(b, B)$-cocycles. 


\subsection{Cyclic cocycles associated to multiple operator integrals}

We define the following Hochschild $n$-cochain:

$$
\phi_{n}\left(a_{0}, \ldots, a_{n}\right):=\left\langle a_{0}\left[D, a_{1}\right],\left[D, a_{2}\right], \ldots,\left[D, a_{n}\right]\right\rangle \quad\left(a_{0}, \ldots, a_{n} \in \mathcal{A}\right) .
$$

We easily see that $B_{0} \phi_{n}$ is invariant under cyclic permutations, so that $B \phi_{n}=n B_{0} \phi_{n}$ for odd $n$ and $B \phi_{n}=0$ for even $n$. Also, $\phi_{n}\left(a_{0}, \ldots, a_{n}\right)=0$ when $a_{j}=1$ for some $j \geq 1$. We put $\phi_{0}:=0$.

Lemma 17. We have $b \phi_{n}=\phi_{n+1}$ for odd $n$ and we have $b \phi_{n}=0$ for even $n$.

Proof. As $b \phi_{0}=0$ by definition, and $b^{2}=0$, we need only check the case in which $n$ is odd.

We find, by splitting up the sum, and shifting the second appearing sum by one, that

$$
\begin{aligned}
b \phi_{n} & \left(a_{0}, \ldots, a_{n+1}\right) \\
= & \left\langle a_{0} a_{1}\left[D, a_{1}\right],\left[D, a_{2}\right], \ldots,\left[D, a_{n+1}\right]\right\rangle-\left\langle a_{0} a_{1}\left[D, a_{1}\right],\left[D, a_{2}\right], \ldots,\left[D, a_{n+1}\right]\right\rangle \\
& +\sum_{j=2}^{n}(-1)^{j}\left\langle a_{0}\left[D, a_{1}\right],\left[D, a_{2}\right], \ldots, a_{j}\left[D, a_{j+1}\right], \ldots,\left[D, a_{n+1}\right]\right\rangle \\
& -\sum_{j=2}^{n+1}(-1)^{j}\left\langle a_{0}\left[D, a_{1}\right],\left[D, a_{2}\right], \ldots,\left[D, a_{j-1}\right] a_{j}, \ldots,\left[D, a_{n+1}\right]\right\rangle \\
& +\left\langle a_{n+1} a_{0}\left[D, a_{1}\right],\left[D, a_{2}\right], \ldots,\left[D, a_{n}\right]\right\rangle \\
= & \sum_{j=2}^{n}(-1)^{j}\left\langle a_{0}\left[D, a_{1}\right],\left[D, a_{2}\right], \ldots,\left[D, a_{n+1}\right]\right\rangle-\left\langle a_{0}\left[D, a_{1}\right],\left[D, a_{2}\right], \ldots,\left[D, a_{n}\right] a_{n+1}\right\rangle \\
& +\left\langle a_{n+1} a_{0}\left[D, a_{1}\right], \ldots,\left[D, a_{n}\right]\right\rangle \\
= & \left\langle\left[D, a_{n+1}\right], a_{0}\left[D, a_{1}\right],\left[D, a_{2}\right], \ldots,\left[D, a_{n}\right]\right\rangle \\
= & \phi_{n+1}\left(a_{0}, \ldots, a_{n+1}\right),
\end{aligned}
$$

by (I) and (II) of Lemma 14 .

Lemma 18. Let $n$ be even. We have $b B_{0} \phi_{n}=2 \phi_{n}-B_{0} \phi_{n+1}$.

Proof. Splitting the sum in two, and shifting the index of the second sum, we find

$$
\begin{aligned}
b B_{0} \phi_{n}\left(a_{0}, \ldots, a_{n}\right)= & \sum_{j=0}^{n-1}(-1)^{j}\left\langle\left[D, a_{0}\right], \ldots, a_{j}\left[D, a_{j+1}\right], \ldots,\left[D, a_{n}\right]\right\rangle \\
& -\sum_{j=1}^{n}(-1)^{j}\left\langle\left[D, a_{0}\right], \ldots,\left[D, a_{j-1}\right] a_{j}, \ldots,\left[D, a_{n}\right]\right\rangle+\left\langle\left[D, a_{n} a_{0}\right], \ldots,\left[D, a_{n-1}\right]\right\rangle \\
= & \left\langle a_{0}\left[D, a_{1}\right],\left[D, a_{2}\right], \ldots,\left[D, a_{n}\right]\right\rangle+\sum_{j=1}^{n-1}(-1)^{j}\left\langle\left[D, a_{0}\right], \ldots,\left[D, a_{n}\right]\right\rangle \\
& -\left\langle\left[D, a_{0}\right], \ldots,\left[D, a_{n-2}\right],\left[D, a_{n-1}\right] a_{n}\right\rangle+\left\langle\left[D, a_{n} a_{0}\right], \ldots,\left[D, a_{n-1}\right]\right\rangle \\
= & \phi_{n}\left(a_{0}, \ldots, a_{n}\right)-\left\langle\left[D, a_{0}\right], \ldots,\left[D, a_{n}\right]\right\rangle+\left\langle\left[D, a_{n}\right],\left[D, a_{0}\right], \ldots,\left[D, a_{n-1}\right]\right\rangle \\
& +\left\langle\left[D, a_{n}\right] a_{0},\left[D, a_{1}\right], \ldots,\left[D, a_{n-1}\right]\right\rangle \\
= & 2 \phi_{n}\left(a_{0}, \ldots, a_{n}\right)-B_{0} \phi_{n+1}\left(a_{0}, \ldots, a_{n}\right),
\end{aligned}
$$

by using both properties of the bracket $\langle\cdot\rangle$ in the last step. 
Motivated by this we define

$$
\psi_{2 k-1}:=\phi_{2 k-1}-\frac{1}{2} B_{0} \phi_{2 k}
$$

so that

$$
B \psi_{2 k+1}=2(2 k+1) b \psi_{2 k-1} .
$$

We can rephrase this property in terms of the $(b, B)$-complex as follows.

Proposition 19. Let $\phi_{n}$ and $\psi_{2 k-1}$ be as defined above and set $\tilde{\psi}_{2 k-1}:=(-1)^{k-1} \frac{(k-1) !}{(2 k-1) !} \psi_{2 k-1}$.

1. The sequence $\left(\phi_{2 k}\right)$ is $a(b, B)$-cocycle and each $\phi_{2 k}$ defines an even Hochschild cocycle: $b \phi_{2 k}=0$.

2. The sequence $\left(\tilde{\psi}_{2 k-1}\right)$ is an odd $(b, B)$-cocycle.

We use an integral notation that is defined by linear extension of

$$
\int_{\phi} a_{0} d a_{1} \cdots d a_{n}:=\int_{\phi_{n}} a_{0} d a_{1} \cdots d a_{n}:=\phi\left(a_{0}, a_{1}, \ldots, a_{n}\right),
$$

and similarly for $\psi$.

\subsection{Derivatives of the spectral action in terms of universal forms}

In this section we will express the derivatives of the fluctuated spectral action (occurring in the Taylor series) in terms of universal forms that are integrated along $\phi$. We thus make the jump from an expression in terms of $V=\pi_{D}(A) \in \Omega_{D}^{1}(\mathcal{A})_{\text {sa }}$ to an expression in terms of $A \in \Omega^{1}(\mathcal{A})$. By $(6)$ and the definition of $\langle V, \ldots, V\rangle$, we have, for $n \in \mathbb{N}$,

$$
\begin{aligned}
\left.\frac{1}{n !} \frac{d^{n}}{d t^{n}} \operatorname{Tr}(f(D+t V))\right|_{t=0} & =\operatorname{Tr}\left(T_{f^{[n]}}^{D}(V, \ldots, V)\right) \\
& =\frac{1}{n}\langle V, \ldots, V\rangle .
\end{aligned}
$$

As $V$ decomposes as a finite sum $V=\sum a_{j}\left[D, b_{j}\right]$, our task is to express $\left\langle a_{j_{1}}\left[D, b_{j_{1}}\right], \ldots, a_{j_{n}}\left[D, b_{j_{n}}\right]\right\rangle$ in terms of universal forms $a_{0} d a_{1} \cdots d a_{n}$ integrated along $\phi$. This will turn out to be possible by just using (II) and $\left[D, a_{1} a_{2}\right]=a_{1}\left[D, a_{2}\right]+\left[D, a_{1}\right] a_{2}$. To find the exact expression we need to work in the algebra $M_{2}\left(\Omega^{\bullet}(\mathcal{A})\right)=M_{2}(\mathbb{C}) \otimes \Omega^{\bullet}(\mathcal{A})$.

Proposition 20. Let $n \in \mathbb{N}$. For $a_{1}, \ldots, a_{n}, b_{1}, \ldots, b_{n} \in \mathcal{A}$, denoting $A_{j}:=a_{j} d b_{j}$, we have

$$
\left\langle a_{1}\left[D, b_{1}\right], \ldots, a_{n}\left[D, b_{n}\right]\right\rangle=\int_{\phi}\left(\begin{array}{ll}
A_{1} & 0
\end{array}\right) \prod_{j=2}^{n}\left(\begin{array}{cc}
A_{j}+d A_{j} & -A_{j} \\
d A_{j} & -A_{j}
\end{array}\right)\left(\begin{array}{l}
1 \\
0
\end{array}\right) .
$$

Proof. If we combine, for every $n \in \mathbb{N}_{0}$, the $n$-multilinear function $\langle\cdot\rangle$ from (12), we obtain a linear function

$$
\langle\cdot\rangle: T \mathcal{B}(\mathcal{H}) \rightarrow \mathbb{C}
$$

on the tensor algebra $T \mathcal{B}(\mathcal{H})$. For any $\omega, v \in T \mathcal{B}(\mathcal{H})$, a straightforward calculation using the commutation rule (II) from Lemma 14 shows that

$$
\left\langle\omega \otimes a_{j-1}\left[D, b_{j-1}\right] \otimes\left(\begin{array}{ll}
a_{j} & a_{j} b_{j}
\end{array}\right) v\right\rangle=\left\langle\omega \otimes\left(a_{j-1} \quad a_{j-1} b_{j-1}\right) M_{j} \otimes v\right\rangle,
$$


where $M_{j} \in M_{2}(T \mathcal{B}(\mathcal{H}))$ is defined by

$$
M_{j}:=\left(\begin{array}{cc}
{\left[D, b_{j-1} a_{j}\right]+\left[D, b_{j-1}\right] \otimes\left[D, a_{j}\right]} & {\left[D, b_{j-1} a_{j} b_{j}\right]+\left[D, b_{j-1}\right] \otimes\left[D, a_{j} b_{j}\right]} \\
-\left[D, a_{j}\right] & -\left[D, a_{j} b_{j}\right]
\end{array}\right) .
$$

Repeating (18), and subsequently using (16), it follows that

$$
\begin{aligned}
& \left\langle a_{1}\left[D, b_{1}\right], \ldots, a_{n}\left[D, b_{n}\right]\right\rangle=\left\langle a_{1}\left[D, b_{1}\right] \otimes \ldots \otimes a_{n-1}\left[D, b_{n-1}\right] \otimes\left(\begin{array}{ll}
a_{n} & a_{n} b_{n}
\end{array}\right)\left(\begin{array}{c}
{\left[D, b_{n}\right.} \\
0
\end{array}\right)\right\rangle \\
& =\left\langle\left(\begin{array}{ll}
a_{1} & a_{1} b_{1}
\end{array}\right)\left(\prod_{j=2}^{n} M_{j}\right)\left(\begin{array}{c}
{\left[D, b_{n}\right.} \\
0
\end{array}\right)\right\rangle \\
& =\int_{\phi}\left(\begin{array}{ll}
a_{1} & a_{1} b_{1}
\end{array}\right)\left(\prod_{j=2}^{n} N_{j}\right)\left(\begin{array}{c}
d b_{n} \\
0
\end{array}\right) \text {, }
\end{aligned}
$$

where from (19) we obtain

$$
\begin{aligned}
N_{j} & =\left(\begin{array}{cc}
d\left(b_{j-1} a_{j}\right)+d b_{j-1} d a_{j} & d\left(b_{j-1} a_{j} b_{j}\right)+d b_{j-1} d\left(a_{j} b_{j}\right) \\
-d a_{j} & -d\left(a_{j} b_{j}\right)
\end{array}\right) \\
& =\left(\begin{array}{cc}
d b_{j-1} & b_{j-1} \\
0 & -1
\end{array}\right)\left(\begin{array}{cc}
a_{j}+d a_{j} & a_{j} b_{j}+d a_{j} b_{j}+a_{j} d b_{j} \\
d a_{j} & d a_{j} b_{j}+a_{j} d b_{j}
\end{array}\right) .
\end{aligned}
$$

By also writing $\left(\begin{array}{c}d b_{n} \\ 0\end{array}\right)=\left(\begin{array}{cc}d b_{n} & b_{n} \\ 0 & -1\end{array}\right)\left(\begin{array}{l}1 \\ 0\end{array}\right)$, we find that

$$
\begin{aligned}
& \left\langle a_{1}\left[D, b_{1}\right], \ldots a_{n}\left[D, b_{n}\right]\right\rangle \\
& =\int_{\phi}\left(\begin{array}{ll}
a_{1} & a_{1} b_{1}
\end{array}\right)\left(\begin{array}{cc}
d b_{1} & b_{1} \\
0 & -1
\end{array}\right)\left(\prod_{j=2}^{n}\left(\begin{array}{cc}
a_{j}+d a_{j} & a_{j} b_{j}+d a_{j} b_{j}+a_{j} d b_{j} \\
d a_{j} & d a_{j} b_{j}+a_{j} d b_{j}
\end{array}\right)\left(\begin{array}{cc}
d b_{j} & b_{j} \\
0 & -1
\end{array}\right)\right)\left(\begin{array}{l}
1 \\
0
\end{array}\right) \\
& =\int_{\phi}\left(\begin{array}{ll}
A_{1} & 0
\end{array}\right)\left(\prod_{j=2}^{n}\left(\begin{array}{cc}
A_{j}+d A_{j} & -A_{j} \\
d A_{j} & -A_{j}
\end{array}\right)\right)\left(\begin{array}{l}
1 \\
0
\end{array}\right),
\end{aligned}
$$

which concludes the proof.

Corollary 21. If $n \in \mathbb{N}, A \in \Omega^{1}(\mathcal{A})$ and $V:=\pi_{D}(A) \in \Omega_{D}^{1}(\mathcal{A})$, then

$$
\langle V, \ldots, V\rangle=\int_{\phi}\left(\begin{array}{ll}
A & 0
\end{array}\right)\left(\begin{array}{cc}
A+d A & -A \\
d A & -A
\end{array}\right)^{n-1}\left(\begin{array}{l}
1 \\
0
\end{array}\right) .
$$

Example 22. Using (20), we obtain in particular

$$
\begin{aligned}
\langle V\rangle & =\int_{\phi_{1}} A, \\
\langle V, V\rangle & =\int_{\phi_{2}} A^{2}+\int_{\phi_{3}} A d A, \\
\langle V, V, V\rangle & =\int_{\phi_{3}} A^{3}+\int_{\phi_{4}} A d A A+\int_{\phi_{5}} A d A d A, \\
\langle V, V, V, V\rangle & =\int_{\phi_{4}} A^{4}+\int_{\phi_{5}}\left(A^{3} d A+A d A A^{2}\right)+\int_{\phi_{6}} A d A d A A+\int_{\phi_{7}} A d A d A d A .
\end{aligned}
$$


With (17), and in the sense of (7), this implies that

$$
\operatorname{Tr}(f(D+V)-f(D))=\int_{\phi_{1}} A+\frac{1}{2} \int_{\phi_{2}} A^{2}+\int_{\phi_{3}}\left(\frac{1}{2} A d A+\frac{1}{3} A^{3}\right)+\int_{\phi_{4}}\left(\frac{1}{3} A d A A+\frac{1}{4} A^{4}\right)+\ldots,
$$

where the dots indicate terms of degree 5 and higher. Using $\phi_{2 k-1}=\psi_{2 k-1}+\frac{1}{2} B_{0} \phi_{2 k}$, this becomes

$$
\begin{aligned}
\operatorname{Tr}(f(D+V)-f(D))= & \int_{\psi_{1}} A+\frac{1}{2} \int_{\phi_{2}}\left(A^{2}+d A\right)+\int_{\psi_{3}}\left(\frac{1}{2} A d A+\frac{1}{3} A^{3}\right) \\
& +\frac{1}{4} \int_{\phi_{4}}\left(d A d A+\frac{2}{3}\left(d A A^{2}+A d A A+A^{2} d A\right)+A^{4}\right)+\ldots
\end{aligned}
$$

Notice that, if $\phi_{4}$ would be tracial, we would be able to identify the terms $d A A^{2}, A d A A$ and $A^{2} d A$, and thus obtain the Yang-Mills form $F^{2}=\left(d A+A^{2}\right)^{2}$, under the fourth integral. In the general case, however, cyclic permutations under $\int_{\phi}$ produce correction terms, of which we will need to keep track.

\subsection{Near-tracial behavior of $\int_{\phi}$}

In $\$ 4.3$. we have not used the cyclicity property (I) from Lemma 14 . Doing so yields the following proposition, which shows how $\int_{\phi}$ differs from being tracial. This proposition and its corollary are crucial for Section 5 .

For a universal $n$-form $X \in \Omega^{n}(\mathcal{A})$, define odd $(X):=1$ if $n$ is odd, and odd $(X):=0$ if $n$ is even.

Proposition 23. Let $X$ and $Y$ be universal forms. Then

$$
\int_{\phi} X Y-\int_{\phi} Y X=\operatorname{odd}(Y) \int_{\phi} Y d X-\operatorname{odd}(X) \int_{\phi} X d Y .
$$

Proof. Without loss of generality, assume that $X=x_{0} d x_{1} \ldots d x_{n}$ and $Y=y_{0} d y_{1} \ldots d y_{k}$ for some $x_{0}, \ldots, x_{n}, y_{0}, \ldots, y_{k} \in \mathcal{A}$. By using $d a b=d(a b)-a d b$ repeatedly, we get

$$
\begin{aligned}
\int_{\phi} X Y= & \int_{\phi} x_{0} d x_{1} \cdots d x_{n-1}\left(d\left(x_{n} y_{0}\right)-x_{n} d y_{0}\right) d y_{1} \cdots d y_{k} \\
= & \int_{\phi} x_{0}\left(d x_{1} \cdots d x_{n-1} d\left(x_{n} y_{0}\right)-d x_{1} \cdots d x_{n-2} d\left(x_{n-1} x_{n}\right) d y_{0}+\ldots\right. \\
& \left.\ldots+(-1)^{n-1} d\left(x_{1} x_{2}\right) d x_{3} \cdots d x_{n} d y_{0}+(-1)^{n} x_{1} d x_{2} \cdots d x_{n} d y_{0}\right) d y_{1} \cdots d y_{k} \\
= & \left\langle x_{0}\left[D, x_{1}\right], \ldots,\left[D, x_{n-1}\right],\left[D, x_{n} y_{0}\right],\left[D, y_{1}\right], \ldots,\left[D, y_{k}\right]\right\rangle \\
& -\left\langle x_{0}\left[D, x_{1}\right], \ldots,\left[D, x_{n-2}\right],\left[D, x_{n-1} x_{n}\right],\left[D, y_{0}\right],\left[D, y_{1}\right], \ldots,\left[D, y_{k}\right]\right\rangle+\ldots \\
& \ldots+(-1)^{n-1}\left\langle x_{0}\left[D, x_{1} x_{2}\right],\left[D, x_{3}\right], \ldots,\left[D, x_{n}\right],\left[D, y_{0}\right],\left[D, y_{1}\right], \ldots,\left[D, y_{k}\right]\right\rangle \\
& +(-1)^{n}\left\langle x_{0} x_{1}\left[D, x_{2}\right],\left[D, x_{3}\right], \ldots,\left[D, x_{n}\right],\left[D, y_{0}\right],\left[D, y_{1}\right], \ldots,\left[D, y_{k}\right]\right\rangle \\
= & \left\langle x_{0}\left[D, x_{1}\right], \ldots,\left[D, x_{n}\right] y_{0},\left[D, y_{1}\right], \ldots,\left[D, y_{k}\right]\right\rangle \\
& +\sum_{j=0}^{n-2}(-1)^{j}\left\langle x_{0}\left[D, x_{1}\right], \ldots,\left[D, x_{n}\right],\left[D, y_{0}\right], \ldots,\left[D, y_{k}\right]\right\rangle \\
= & \left\langle x_{0}\left[D, x_{1}\right], \ldots,\left[D, x_{n}\right], y_{0}\left[D, y_{1}\right], \ldots,\left[D, y_{k}\right]\right\rangle-\operatorname{odd}(X) \int_{\phi} X d Y .
\end{aligned}
$$


Doing the same for $\int_{\phi} Y X$ and using cyclicity (Lemma 14) yields the proposition.

A quick check shows that the above proposition implies the following handy rules.

Corollary 24. Let $X, Y \in \Omega^{\bullet}(\mathcal{A})$, and $A \in \Omega^{1}(\mathcal{A})$.

(i) If $X$ and $Y$ are both of even degree, then

$$
\int_{\phi} X Y=\int_{\phi} Y X
$$

(ii) If $X$ has odd degree, then

$$
\int_{\phi}(A X-X A)=\int_{\phi} d(A X)
$$

(iii) If $X$ has even degree, then

$$
\int_{\phi}(X A-A X)=\int_{\phi} d X A+\int_{\phi} d A d X
$$

\subsection{Higher-order generalized Chern-Simons forms}

As a final preparation for our main result, we briefly recall from [21] the definition of ChernSimons forms.

Definition 25. Let $\left(\Omega^{\bullet}, d\right)$ be a differential graded algebra. The Chern-Simons form of degree $2 k-1$ is given for $A \in \Omega^{1}$ by

$$
\operatorname{cs}_{2 k-1}(A):=\int_{0}^{1} A\left(F_{t}\right)^{k-1} d t,
$$

where $F_{t}=t d A+t^{2} A^{2}$ is the curvature two-form of the (connection) one-form $A_{t}=t A$. $\mathcal{A}$.

We will only work with the universal differential graded algebra $\Omega^{\bullet}=\Omega^{\bullet}(\mathcal{A})$ for the algebra

Example 26. For the first three Chern-Simons forms one easily derives the following explicit expressions:

$$
\begin{gathered}
\operatorname{cs}_{1}(A)=A ; \quad \operatorname{cs}_{3}(A)=\frac{1}{2}\left(A d A+\frac{2}{3} A^{3}\right) \\
\operatorname{cs}_{5}(A)=\frac{1}{3}\left(A(d A)^{2}+\frac{3}{4} A d A A^{2}+\frac{3}{4} A^{3} d A+\frac{3}{5} A^{5}\right) .
\end{gathered}
$$

These are well-known expressions from the physics literature, cf. [18, Section 11.5.2]. 


\section{Expansion of the spectral action in terms of $(b, B)$-cocycles}

In this section we prove our main theorem.

Theorem 27. Let $(\mathcal{A}, \mathcal{H}, D)$ be an s-summable spectral triple, and let $f \in \mathcal{E}^{s, \gamma}$ for $\gamma \in(0,1)$. The spectral action fluctuated by $V=\pi_{D}(A) \in \Omega_{D}^{1}(\mathcal{A})_{\text {sa }}$ can be written as

$$
\operatorname{Tr}(f(D+V)-f(D))=\sum_{k=1}^{\infty}\left(\int_{\psi_{2 k-1}} \operatorname{cs}_{2 k-1}(A)+\frac{1}{2 k} \int_{\phi_{2 k}} F^{k}\right),
$$

where the series converges absolutely.

We prove this theorem in two steps. Firstly, we deal with the algebraic part of this statement, in \$5.1. Here we only need to assume $f \in \mathcal{W}^{s, n}$ for a finite $n \in \mathbb{N}$. Secondly, in 85.2 , we tackle the analytical part. We there obtain a strong estimate on the remainder of the above expansion in Theorem 33 for a function $f \in \mathcal{E}^{s, \gamma}$ for general $\gamma \in(0,1]$. This estimate will imply that the conclusion of Theorem 27 still holds in the case of $\gamma=1$, when the perturbation $V$ is sufficiently small. When $f \in \mathcal{E}^{s, \gamma}$ for $\gamma \in(0,1)$, the expansion follows for all perturbations, and thus we prove Theorem 27

\subsection{Truncated expansion}

Let $K \in \mathbb{N}, f \in \mathcal{W}^{s, 2 K}$, and $V=\pi_{D}(A) \in \Omega_{D}^{1}(\mathcal{A})_{\text {sa. }}$. We prove a truncated version of Theorem 27 . showing that the fluctuation of the spectral action can be expressed in terms of Chern-Simons and Yang-Mills forms, up to a remainder which involves forms of degree higher than $K$. To enumerate the remainder forms we use the index set

$$
T_{K}:=\left\{(v, w, p) \in \coprod_{m \in \mathbb{N}_{0}}\left(\mathbb{N}_{0} \times \mathbb{N}^{m-1}\right) \times \mathbb{N}^{m} \times \mathbb{N}_{0}|| v|+| w\left|+\left\lfloor\frac{p}{2}\right\rfloor<K, 2\right| v|+| w \mid+p \geq K\right\} .
$$

Proposition 28. We have the asymptotic expansion

$$
\operatorname{Tr}(f(D+V)-f(D)) \sim \sum_{k=1}^{\infty} \int_{\phi}\left(\operatorname{cs}_{2 k-1}(A)+\int_{0}^{1} A F_{t}^{k-1} t A d t\right)
$$

by which we mean that we can write the $K^{\text {th }}$ remainder of this expansion as

$$
\begin{aligned}
& \operatorname{Tr}(f(D+V)-f(D))-\sum_{k=1}^{K} \int_{\phi}\left(\operatorname{cs}_{2 k-1}(A)+\int_{0}^{1} A F_{t}^{k-1} t A d t\right) \\
& \quad=\operatorname{Tr}\left(T_{f^{[K+1]}}^{D+V, D, \ldots, D}(V, \ldots, V)\right)-\sum_{(v, w, p) \in T_{K}} \frac{1}{2|v|+|w|+p+1} \int_{\phi} A A^{2 v_{1}}(d A)^{w_{1}} \cdots A^{2 v_{m}}(d A)^{w_{m}} A^{p},
\end{aligned}
$$

where $T_{K}$, defined by (22), satisfies $\left|T_{K}\right| \leq 2^{K+1}$, and where $f \in \mathcal{W}^{s, 2 K}$. 
Proof. We start with the 2x2 matrix equation from Corollary 21 and separate the 1-forms $A$ from the two-forms $d A$. The $n$-th term in the Taylor expansion of $\operatorname{Tr}(f(D+V))$ is given (by use of (17) and Corollary 21, by

$$
\begin{aligned}
\left.\frac{1}{n !} \frac{d^{n}}{d t^{n}} \operatorname{Tr}(f(D+t V))\right|_{t=0} & =\frac{1}{n} \int_{\phi}\left(\begin{array}{ll}
A & 0
\end{array}\right)\left(\left(\begin{array}{cc}
A & -A \\
0 & -A
\end{array}\right)+\left(\begin{array}{ll}
d A & 0 \\
d A & 0
\end{array}\right)\right)^{n-1}\left(\begin{array}{l}
1 \\
0
\end{array}\right) \\
& \equiv \frac{1}{n} \int_{\phi}\left(\begin{array}{ll}
A & 0
\end{array}\right)(\alpha A+\beta d A)^{n-1}\left(\begin{array}{l}
1 \\
0
\end{array}\right) \\
& =\frac{1}{n} \int_{\phi} A e_{1}^{t}(\alpha A+\beta d A)^{n-1} e_{1} .
\end{aligned}
$$

for some scalar-valued $2 \times 2$ matrices $\alpha$ and $\beta$, and $e_{1}=\left(\begin{array}{l}1 \\ 0\end{array}\right)$. The $\alpha^{\prime}$ s and $\beta^{\prime}$ s have very nice algebraic properties, which can be used to regroup the terms in the expansion in $n$. When summing 23) from $n=1$ to infinity, and grouping the universal forms by their degree as in Example 22. we need some machinery to keep track of the coefficient $1 / n$. We will work in the space of (finite) polynomials $M_{2}\left(\Omega^{\bullet}(\mathcal{A})\right)[t]$, and define an integration with respect to $t$ as the linear map $\int_{0}^{1} d t: M_{2}\left(\Omega^{\bullet}(\mathcal{A})\right)[t] \rightarrow M_{2}\left(\Omega^{\bullet}(\mathcal{A})\right)$ given by integration of polynomials. We thus obtain

$$
\begin{aligned}
\left.\frac{1}{n !} \frac{d^{n}}{d t^{n}} \operatorname{Tr}(f(D+t V))\right|_{t=0} & =\int_{0}^{1} d t t^{n-1} \int_{\phi} A e_{1}^{t}(\alpha A+\beta d A)^{n-1} e_{1} \\
& =\int_{0}^{1} d t \int_{\phi} A e_{1}^{t}(\alpha t A+\beta t d A)^{n-1} e_{1} .
\end{aligned}
$$

We now expand the $(n-1)$-th power, which is complicated because $\alpha$ and $\beta$ do not commute. To avoid notational clutter, let us denote $X:=t A$ and $Y:=t d A$. We find

$$
e_{1}^{t}(\alpha X+\beta Y)^{n-1} e_{1}=\sum_{\substack{k=0 \\ v_{1} \geq 0, v_{2}, \ldots, v_{k} \geq 1 \\ w_{1}, \ldots, w_{k} \geq 1, p \geq 0 \\|v|+|w|+p=n-1}}^{\left\lceil\frac{n-1}{2}\right\rceil} e_{1}^{t}\left(\alpha^{v_{1}} \beta^{w_{1}} \cdots \alpha^{v_{k}} \beta^{w_{k}} \alpha^{p}\right) e_{1} X^{v_{1}} Y^{w_{1}} \cdots X^{v_{k}} Y^{w_{k}} X^{p}
$$

We can summarize the identities involving $\alpha$ and $\beta$ that we will use as

$$
\begin{aligned}
& \alpha^{2}=1 ; \quad \beta^{2}=\beta ; \quad \beta \alpha \beta=0 ; \quad e_{1}^{t}(\alpha) e_{1}=1 ; \\
& e_{1}^{t}(\alpha \beta \alpha) e_{1}=0 ; \quad e_{1}^{t}(\alpha \beta) e_{1}=0 ; \quad e_{1}^{t}(\beta \alpha) e_{1}=1 ; \quad e_{1}^{t}(\beta) e_{1}=1 .
\end{aligned}
$$

From these identities follow the following two remarks:

- If $k \geq 2$ and $v_{i}$ is odd for a certain $i \in\{2, \ldots, k\}$, then somewhere in $\alpha^{v_{1}} \beta^{w_{1}} \cdots \alpha^{v_{k}} \beta^{w_{k}} \alpha^{p}$ a factor $\beta \alpha \beta=0$ occurs, so in particular

$$
e_{1}^{t}\left(\alpha^{v_{1}} \beta^{w_{1}} \cdots \alpha^{v_{k}} \beta^{w_{k}} \alpha^{p}\right) e_{1}=0
$$

- If $v_{1}$ is odd and $v_{2}, \ldots, v_{k}$ are all even, then

$$
e_{1}^{t}\left(\alpha^{v_{1}} \beta^{w_{1}} \cdots \alpha^{v_{k}} \beta^{w_{k}} \alpha^{p}\right) e_{1}=e_{1}^{t}\left(\alpha \beta \alpha^{p}\right) e_{1}=0 .
$$


Therefore, for all $k \geq 0$, we conclude that in (25) only terms remain in which $v_{1}, \ldots, v_{k}$ are even. In fact, we find

$$
\begin{aligned}
& e_{1}^{t}(\alpha X+\beta Y)^{n-1} e_{1}=\sum_{k=0}^{\left\lceil\frac{n-1}{2}\right\rceil} \sum_{\begin{array}{c}
v_{1} \in 2 \mathbb{N}_{0}, v_{2}, \ldots, v_{k} \in 2 \mathbb{N} \\
w_{1} \ldots, w_{k} \geq 1, p \geq 0 \\
|v|+|w|+p=n-1
\end{array}} e_{1}^{t}\left(\alpha^{v_{1}} \beta^{w_{1}} \cdots \alpha^{v_{k}} \beta^{w_{k}} \alpha^{p}\right) e_{1} X^{v_{1}} Y^{w_{1}} \cdots X^{v_{k}} Y^{w_{k}} X^{p} \\
& =\sum_{k=0}^{\left\lceil\frac{n-1}{2}\right\rfloor} \sum_{\substack{v_{1} \in 2 \mathbb{N}_{0}, v_{2}, \ldots, v_{k} \in 2 \mathbb{N} \\
w_{1}, \ldots, w_{k} \geq 1, p \geq 0 \\
|v|+|w|+p=n-1}} e_{1}^{t}\left(\beta \alpha^{p}\right) e_{1} X^{v_{1}} Y^{w_{1}} \cdots X^{v_{k}} Y^{w_{k}} X^{p} \\
& =\sum_{k=0}^{\left\lceil\frac{n-1}{2}\right\rceil} \sum_{\begin{array}{c}
v_{1} \geq 0, v_{2} \ldots, v_{k} \geq 1, \\
w_{1}, \ldots, w_{k} \geq 1, p \geq 0, \\
2|v|+|w|+p=n-1
\end{array}}\left(X^{2}\right)^{v_{1}} Y^{w_{1}} \cdots\left(X^{2}\right)^{v_{k}} Y^{w_{k}} X^{p} .
\end{aligned}
$$

Summing this from $n=1$ to $K$, we can write

$$
\sum_{n=1}^{K} e_{1}^{t}(\alpha X+\beta Y)^{n-1} e_{1}=\sum_{(v, w, p) \in P_{K}}\left(X^{2}\right)^{v_{1}} Y^{w_{1}} \cdots\left(X^{2}\right)^{v_{m}} Y^{w_{m}} X^{p},
$$

where $P_{K}$ is the set of $(v, w, p) \in \coprod_{m}\left(\mathbb{N}_{0} \times \mathbb{N}^{m-1}\right) \times \mathbb{N}^{m} \times \mathbb{N}_{0}$ such that $2|v|+w+p<K$. In this last expression we can almost recognize an expansion of $\left(X^{2}+Y\right)^{k-1}=F_{t}^{k-1}$. Indeed, we have

$$
\sum_{k=1}^{K}\left(X^{2}+Y\right)^{k-1}(1+X)=\sum_{(v, w, p) \in S_{K}}\left(X^{2}\right)^{v_{1}} Y^{w_{1}} \cdots\left(X^{2}\right)^{v_{m}} Y^{w_{m}} X^{p},
$$

where $S_{K}$ is the set of $(v, w, p) \in \coprod_{m}\left(\mathbb{N}_{0} \times \mathbb{N}^{m-1}\right) \times \mathbb{N}^{m} \times \mathbb{N}_{0}$ such that $|v|+|w|+\left\lfloor\frac{p}{2}\right\rfloor<K$. By (27) we have $\left|S_{K}\right| \leq 2^{K+1}$. By using $T_{K}=S_{K} \backslash P_{K}$, we can combine (24), (26) and (27), and obtain

$$
\begin{aligned}
\sum_{n=1}^{K} & \left.\frac{1}{n !} \frac{d^{n}}{d t^{n}} \operatorname{Tr}(f(D+t V))\right|_{t=0}-\sum_{k=1}^{K} \int_{\phi} \int_{0}^{1} A F_{t}^{k-1}(1+t A) d t \\
& =-\sum_{(v, w, p) \in T_{K}} \frac{1}{2|v|+|w|+p+1} \int_{\phi} A A^{2 v_{1}}(d A)^{w_{1}} \cdots A^{2 v_{m}}(d A)^{w_{m}} A^{p} .
\end{aligned}
$$

Together with (8), and the definition (21) of $\operatorname{cs}_{2 k-1}(A),(28)$ implies the proposition.

Theorem 29. We have

$$
\int_{\phi}\left(\operatorname{cs}_{2 k-1}(A)+\int_{0}^{1} A F_{t}^{k-1} t A d t\right)=\int_{\psi_{2 k-1}} \operatorname{cs}_{2 k-1}(A)+\frac{1}{2 k} \int_{\phi_{2 k}} F^{k} .
$$

Therefore, with the same remainder term as in Proposition 28, we have

$$
\operatorname{Tr}(f(D+V)-f(D)) \sim \sum_{k=1}^{\infty}\left(\int_{\psi_{2 k-1}} \operatorname{cs}_{2 k-1}(A)+\frac{1}{2 k} \int_{\phi_{2 k}} F^{k}\right) .
$$


The Chern-Simons term in Proposition 28 , integrated along $\phi$, yields the correct Chern-Simons term integrated along $\psi$, plus an additional term. Indeed, recall that $\phi_{2 k-1}=\psi_{2 k-1}+\frac{1}{2} B_{0} \phi_{2 k}$ so that we find

$$
\begin{aligned}
\int_{\phi_{2 k-1}} A F_{t}^{k-1}+\int_{\phi_{2 k-1}} t A F_{t}^{k-1} A & =\int_{\psi_{2 k-1}} A F_{t}^{k-1}+\int_{\phi_{2 k}}\left(\frac{1}{2} d\left(A F_{t}^{k-1}\right)+t A F_{t}^{k-1} A\right) \\
& =\int_{\psi_{2 k-1}} A F_{t}^{k-1}+\frac{1}{2} \int_{\phi_{2 k}}\left(d A F_{t}^{k-1}+t A^{2} F_{t}^{k-1}+t A F_{t}^{k-1} A\right),
\end{aligned}
$$

where we used the repeated Bianchi identity $d\left(F_{t}^{k-1}\right)=-\left[A_{t}, F_{t}^{k-1}\right]$ in going to the last line.

We arrive at the following formula:

$$
\operatorname{Tr}(f(D+V)-f(D)) \sim \sum_{k=1}^{\infty}\left(\int_{\psi_{2 k-1}} \operatorname{cs}_{2 k-1}(A)+\frac{1}{2} \int_{0}^{1} d t \int_{\phi_{2 k}}\left(d A F_{t}^{k-1}+t A^{2} F_{t}^{k-1}+t A F_{t}^{k-1} A\right)\right) .
$$

We are now to show that the second term, namely

$$
\begin{aligned}
Y M_{k} & :=\frac{1}{2} \int_{0}^{1} d t \int_{\phi_{2 k}}\left(d A F_{t}^{k-1}+t A^{2} F_{t}^{k-1}+t A F_{t}^{k-1} A\right) \\
& =\int_{0}^{1} d t \frac{1}{2 t} \int_{\phi_{2 k}}\left(d A_{t} F_{t}^{k-1}+A_{t}^{2} F_{t}^{k-1}+A_{t} F_{t}^{k-1} A_{t}\right),
\end{aligned}
$$

equals $\frac{1}{2 k} \int_{\phi_{2 k}} F^{k}$. After some rearrangement we can use Corollary 24 (ii) to find

$$
\begin{aligned}
Y M_{k} & =\int_{0}^{1} d t \frac{1}{2 t} \int_{\phi_{2 k}}\left(d A_{t}+2 A_{t}^{2}\right) F_{t}^{k-1}+\int_{0}^{1} d t \frac{1}{2 t} \int_{\phi_{2 k}}\left(A_{t} F_{t}^{k-1} A_{t}-A_{t}^{2} F_{t}^{k-1}\right) \\
& =\int_{0}^{1} d t \frac{1}{2 t} \int_{\phi_{2 k}}\left(d A_{t}+2 A_{t}^{2}\right) F_{t}^{k-1}-\int_{0}^{1} d t \frac{1}{2 t} \int_{\phi_{2 k+1}} d\left(A_{t}^{2} F_{t}^{k-1}\right) .
\end{aligned}
$$

We will first show that the second term of (29) vanishes. We use the following rule, which allows us to replace the integrand by a form which is two degrees lower.

Lemma 30. For every $m \geq 0$, we have

$$
\int_{\phi_{2 m+3}} d\left(A_{t}^{2} F_{t}^{m}\right)=-\int_{\phi_{2 m+1}}\left(d\left(A_{t}^{2} F_{t}^{m-1}\right)+d A_{t} d\left(F_{t}^{m-1}\right)\right) .
$$

Proof. We use the definition of $F_{t}$, the repeated Bianchi identity $d\left(F_{t}^{m}\right)=\left[F_{t}^{m}, A_{t}\right]$, and subsequently Proposition 23, to obtain

$$
\begin{aligned}
\int_{\phi_{2 m+1}} d\left(A_{t}^{2} F_{t}^{m-1}\right) & =\int_{\phi_{2 m+1}}\left(d\left(F_{t} F_{t}^{m-1}\right)-d\left(d A_{t} F_{t}^{m-1}\right)\right) \\
& =\int_{\phi_{2 m+1}}\left(d\left(F_{t}^{m}\right)-d A_{t} d\left(F_{t}^{m-1}\right)\right) \\
& =\int_{\phi_{2 m+1}}\left(F_{t}^{m} A_{t}-A_{t} F_{t}^{m}\right)-\int_{\phi_{2 m+1}} d A_{t} d\left(F_{t}^{m-1}\right) \\
& =\int_{\phi_{2 m+2}} A_{t} d\left(F_{t}^{m}\right)-\int_{\phi_{2 m+1}} d A_{t} d\left(F_{t}^{m-1}\right) \\
& =\int_{\phi_{2 m+2}}\left(A_{t} F_{t}^{m} A_{t}-A_{t}^{2} F_{t}^{m}\right)-\int_{\phi_{2 m+1}} d A_{t} d\left(F_{t}^{m-1}\right) .
\end{aligned}
$$


Applying Corollary 24(ii) to the first term gives

$$
\int_{\phi_{2 m+1}} d\left(A_{t}^{2} F_{t}^{m-1}\right)=-\int_{\phi_{2 m+3}} d\left(A_{t}^{2} F_{t}^{m}\right)-\int_{\phi_{2 m+1}} d A_{t} d\left(F_{t}^{m-1}\right),
$$

which implies the lemma.

We obtain the following result.

Lemma 31. For every $m \geq 0$, we have

$$
\int_{\phi_{2 m+3}} d\left(A_{t}^{2} F_{t}^{m}\right)=0
$$

Proof. This is easily checked when $m=0$, and when $m=1$, it follows from Lemma 30. If $m \geq 2$, we apply Lemma 30 twice, and find

$$
\begin{aligned}
\int_{\phi_{2 m+3}} d\left(A_{t}^{2} F_{t}^{m}\right) & =-\int_{\phi_{2 m+1}} d\left(A_{t}^{2} F_{t}^{m-1}\right)-\int_{\phi_{2 m+1}} d A_{t} d\left(F_{t}^{m-1}\right) \\
& =\int_{\phi_{2 m-1}} d\left(A_{t}^{2} F_{t}^{m-2}\right)+\int_{\phi_{2 m-1}} d A_{t} d\left(F_{t}^{m-2}\right)-\int_{\phi_{2 m+1}} d A_{t} d\left(F_{t}^{m-1}\right) .
\end{aligned}
$$

We recognize $F_{t}=A_{t}^{2}+d A_{t}$ in the first two terms above, so by the Bianchi identity we obtain

$$
\begin{aligned}
\int_{\phi_{2 m+3}} d\left(A_{t}^{2} F_{t}^{m}\right) & =\int_{\phi_{2 m-1}} d\left(F_{t}^{m-1}\right)-\int_{\phi_{2 m+1}} d A_{t} d\left(F_{t}^{m-1}\right) \\
& =\int_{\phi_{2 m-1}}\left(F_{t}^{m-1} A_{t}-A_{t} F_{t}^{m-1}\right)-\int_{\phi_{2 m+1}} d A_{t} d\left(F_{t}^{m-1}\right) .
\end{aligned}
$$

By Corollary 24(iii), the Bianchi identity, and Corollary 24](i), this gives

$$
\begin{aligned}
\int_{\phi_{2 m+3}} d\left(A_{t}^{2} F_{t}^{m}\right) & =\int_{\phi_{2 m}} d\left(F_{t}^{m-1}\right) A_{t} \\
& =\int_{\phi_{2 m}}\left(F_{t}^{m-1} A_{t}^{2}-A_{t} F_{t}^{m-1} A_{t}\right) \\
& =\int_{\phi_{2 m}}\left(A_{t}^{2} F_{t}^{m-1}-A_{t} F_{t}^{m-1} A_{t}\right) .
\end{aligned}
$$

We apply Corollary 24](ii), to find

$$
\int_{\phi_{2 m+3}} d\left(A_{t}^{2} F_{t}^{m}\right)=\int_{\phi_{2 m+1}} d\left(A_{t}^{2} F_{t}^{m-1}\right)
$$

By induction, it follows that $\int_{\phi} d\left(A_{t}^{2} F_{t}^{m}\right)=0$ for all $m$.

By the above Lemma, only the first term of 29] remains, namely,

$$
Y M_{k}=\int_{0}^{1} d t \frac{1}{2 t} \int_{\phi_{2 k}}\left(d A_{t}+2 A_{t}^{2}\right) F_{t}^{k-1}=\int_{0}^{1} d t \int_{\phi_{2 k}}\left(\frac{1}{2} d A+t A^{2}\right) F_{t}^{k-1} .
$$

To express $Y M_{k}$ in an even simpler form, we now remove the integral over $t$, which is possible by the following lemma. 
Lemma 32. We have

$$
\int_{0}^{1} d t \int_{\phi_{2 k}}\left(\frac{1}{2} d A+t A^{2}\right) F_{t}^{k-1}=\frac{1}{2 k} \int_{\phi_{2 k}} F^{k}
$$

Proof. Recall that $\Omega^{\bullet}(\mathcal{A})[t]$ is the space of polynomials with coefficients in the algebra $\Omega^{\bullet}(\mathcal{A})$. The linear map $\frac{d}{d t}: \Omega^{\bullet}(\mathcal{A})[t] \rightarrow \Omega^{\bullet}(\mathcal{A})[t]$ is defined by $\frac{d}{d t}\left(t^{n} B\right):=n t^{n-1} B$ for $B \in \Omega^{\bullet}(\mathcal{A})$, and satisfies the Leibniz rule. Therefore,

$$
\frac{d}{d t}\left(F_{t}^{k}\right)=\frac{d}{d t}\left(F_{t}\right) F_{t}^{k-1}+F_{t} \frac{d}{d t}\left(F_{t}\right) F_{t}^{k-2}+\ldots+F_{t}^{k-1} \frac{d}{d t}\left(F_{t}\right)
$$

Both $F_{t}$ and $\frac{d}{d t}\left(F_{t}\right)$ are 2-forms, so, after a few applications of Corollary 24(i), we arrive at

$$
\int_{\phi_{2 k}} \frac{d}{d t}\left(F_{t}^{k}\right)=k \int_{\phi_{2 k}} \frac{d}{d t}\left(F_{t}\right) F_{t}^{k-1}=k \int_{\phi_{2 k}}\left(d A+2 t A^{2}\right) F_{t}^{k-1}
$$

The fundamental theorem of calculus (for polynomials) gives

$$
\int_{0}^{1} d t \int_{\phi_{2 k}}\left(d A+2 t A^{2}\right) F_{t}^{k-1}=\frac{1}{k} \int_{\phi_{2 k}} \int_{0}^{1} d t \frac{d}{d t}\left(F_{t}^{k}\right)=\frac{1}{k} \int_{\phi_{2 k}}\left(F_{1}^{k}-F_{0}^{k}\right)=\frac{1}{k} \int_{\phi_{2 k}} F^{k},
$$

from which the lemma follows.

Proof of Theorem 29. Applying Lemma 32 to our earlier expression for $Y M_{k}$ (equation (30), we find that

$$
Y M_{k}=\frac{1}{2 k} \int_{\phi_{2 k}} F^{k}
$$

We therefore obtain the theorem.

\subsection{Convergence}

We prove a strong bound on the asymptotic expansion given by Theorem 29 , in particular giving sufficient conditions for the series to converge, effectively replacing $\sim$ by $=$. A crucial ingredient is Lemma 7 .

Theorem 33. Let $(\mathcal{A}, \mathcal{H}, D)$ be an s-summable spectral triple, let $n \in \mathbb{N}$, and fix $f \in \mathcal{E}^{s, \gamma}$ for $\gamma \in$ $(0,1]$. Then there exists $C_{f, s, n, \gamma}$ such that, for $A=\sum_{j=1}^{n} a_{j} d b_{j}$ and $V=\sum_{j=1}^{n} a_{j}\left[D, b_{j}\right]$ self-adjoint with $\left\|a_{j}\right\|,\left\|b_{j}\right\|,\left\|\left[D, a_{j}\right]\right\|,\left\|\left[D, b_{j}\right]\right\| \leq R$, we have

$$
\begin{aligned}
& \left|\operatorname{Tr}(f(D+V)-f(D))-\sum_{k=1}^{K}\left(\int_{\psi_{2 k-1}} \operatorname{cs}_{2 k-1}(A)+\frac{1}{2 k} \int_{\phi_{2 k}} F^{k}\right)\right| \\
& \quad \leq \frac{C_{f, s, n, \gamma}^{K+1}}{K !^{1-\gamma}} \max \left(R^{2 K+2}, R^{4 K+2+4 s}\right) \operatorname{Tr}\left|(D-i)^{-s}\right|,
\end{aligned}
$$

for all $K \in \mathbb{N}_{0}$. Moreover, we have

$$
\left|\int_{\psi_{2 k-1}} \operatorname{cs}_{2 k-1}(A)\right|+\left|\int_{\phi_{2 k}} F^{k}\right| \leq \frac{C_{f, s, n, \gamma}^{k}}{k !^{1-\gamma}} \max \left(R^{2 k}, R^{4 k}\right) \operatorname{Tr}\left|(D-i)^{-s}\right| .
$$


Proof. Theorem 29 gives

$$
\begin{aligned}
& \left|\operatorname{Tr}(f(D+V)-f(D))-\sum_{k=1}^{K}\left(\int_{\psi_{2 k-1}} \operatorname{cs}_{2 k-1}(A)+\frac{1}{2 k} \int_{\phi_{2 k}} F^{k}\right)\right| \\
& \quad \leq\left\|T_{f^{[K+1]}}^{D+V, D, \ldots, D}(V, \ldots, V)\right\|_{1}+\sum_{(v, w, p) \in T_{K}}\left|\int_{\phi} A A^{2 v_{1}}(d A)^{w_{1}} \cdots A^{2 v_{m}}(d A)^{w_{m}} A^{p}\right| .
\end{aligned}
$$

We first focus on the first term. Lemma 7 gives a $C \geq 1$ such that

$$
\begin{aligned}
\left\|T_{f^{[K+1]}}^{D+V, D, \ldots, D}(V, \ldots, V)\right\|_{1} & \leq \frac{C^{K+1}}{K !^{1-\gamma}} \sum_{j_{1}, \ldots, j_{K+1} \in\{1, \ldots, n\}} \prod_{m=1}^{K+1}\left\|a_{j_{m}}\right\|\left\|\left[D, b_{j_{m}}\right]\right\|(1+\|V\|)^{2 s} \operatorname{Tr}\left|(D-i)^{-s}\right| \\
& \leq \frac{C^{K+1}}{K !^{1-\gamma}} n^{K+1} R^{2 K+2}(1+\|V\|)^{2 s} \operatorname{Tr}\left|(D-i)^{-s}\right|,
\end{aligned}
$$

for all $K \in \mathbb{N}_{0}$. We conclude that there exists $\tilde{C}_{f, s, n, \gamma}$ such that

$$
\left\|T_{f^{[K+2]}}^{D+V, D, \ldots, D}(V, \ldots, V)\right\|_{1} \leq \frac{\tilde{C}_{f, s, n, \gamma}^{K+1}}{K !^{1-\gamma}} \max \left(R^{2 K+2}, R^{2 K+2+4 s}\right) \operatorname{Tr}\left|(D-i)^{-s}\right| .
$$

We now move on to the second term (the finite sum) on the right-hand side of (32). It contains terms of the form

$$
\left|\int_{\phi} B_{1} \cdots B_{M}\right|
$$

for $B_{1}, \ldots, B_{M} \in\left\{a_{j} d b_{j}, d a_{j} d b_{j}: j \in\{1, \ldots, n\}\right\}$. Let $l$ be the degree of $B_{1} \cdots B_{M}$. By the definition of $T_{K}$ (equation (22)) $K+1 \leq l \leq 2 K+1$ and $K+1 \leq M \leq 2 K+1$. By using the Leibniz rule repeatedly, we can write

$$
\int_{\phi} B_{1} \cdots B_{M}=\sum_{j \in J} \int_{\phi} e_{0, j} d e_{1, j} \cdots d e_{l, j}
$$

for a set $J$ with $|J| \leq 3^{M} \leq 3^{2 K+1}$, and $e_{i, j} \in \mathcal{A}$ such that $e_{0, j} \cdots e_{l, j}=\prod_{m=1}^{M} a_{j_{m}} b_{j_{m}}$. We get

$$
\begin{aligned}
\left|\int_{\phi} B_{1} \cdots B_{M}\right| & \leq \sum_{j \in J}\left|\int_{\phi} e_{0, j} d e_{1, j} \cdots d e_{l, j}\right| \\
& =\sum_{j \in J}\left|\phi_{l}\left(e_{0, j}, \ldots, e_{l, j}\right)\right| \\
& \leq \sum_{j \in J} \sum_{i=1}^{l} \mid \operatorname{Tr}\left(T_{f^{[l]}}^{D}\left(\left[D, e_{i}\right], \ldots,\left[D, e_{l}\right], e_{0}\left[D, e_{1}\right],\left[D, e_{2}\right], \ldots,\left[D, e_{i-1}\right]\right) \mid,\right.
\end{aligned}
$$

where we suppressed the index $j$ for readability. We now apply Lemma 7 with $V=0$ to (33) and obtain

$$
\left|\int_{\phi} B_{1} \cdots B_{M}\right| \leq l C^{l+1} l !^{\gamma-1} \sum_{j \in J}\left\|e_{0}\right\|\left(\prod_{i=1}^{l}\left\|\left[D, e_{i}\right]\right\|\right) \operatorname{Tr}\left|(D-i)^{-s}\right|,
$$


for a constant $C \geq 1$. Because we have $\left\|a_{j}\right\|,\left\|b_{j}\right\|,\left\|\left[D, a_{j}\right]\right\|,\left\|\left[D, b_{j}\right]\right\| \leq R$ by assumption, and $e_{0} \cdots e_{l}=\prod_{m=1}^{M} a_{j_{m}} b_{j_{m}}$, with $K+1 \leq M \leq 2 K+1$, we find

$$
\begin{aligned}
\left|\int_{\phi} B_{1} \cdots B_{M}\right| & \leq \tilde{C}^{l+1} l !^{\gamma-1} \sum_{j \in J} R^{2 M} \operatorname{Tr}\left|(D-i)^{-s}\right| \\
& \leq \hat{C}^{K+1}|J| K !^{\gamma-1} \max \left(R^{2 K+2}, R^{4 K+2}\right) \operatorname{Tr}\left|(D-i)^{-s}\right| \\
& \leq \check{C}^{K+1} K !^{\gamma-1} \max \left(R^{2 K+2}, R^{4 K+2}\right) \operatorname{Tr}\left|(D-i)^{-s}\right| .
\end{aligned}
$$

We can now bound the second term on the right-hand side of $(32)$. We use that $\left|T_{K}\right| \leq 2^{K+1}$, and that $n^{M} \leq\left(n^{2}\right)^{K+1}$, to find

$$
\begin{aligned}
& \sum_{(v, w, p) \in T_{K}}\left|\int_{\phi} A A^{2 v_{1}}(d A)^{w_{1}} \cdots A^{2 v_{m}}(d A)^{w_{m}} A^{p}\right| \\
& \leq 2^{K+1}\left(n^{2}\right)^{K+1} \check{C}^{K+1} K !^{\gamma-1} \max \left(R^{2 K+2}, R^{4 K+2}\right) \operatorname{Tr}\left|(D-i)^{-s}\right| \\
& \leq \breve{C}_{f, s, n, \gamma}^{K+1} K !^{\gamma-1} \max \left(R^{2 K+2}, R^{4 K+2}\right) \operatorname{Tr}\left|(D-i)^{-s}\right| .
\end{aligned}
$$

Combining the first and second term of (32), we obtain a number $C_{f, s, n, \gamma}$ such that (31) holds.

Moving on to the last claim of the theorem, we notice that, because $\psi_{2 k-1}=\phi_{2 k-1}-\frac{1}{2} B_{0} \phi_{2 k}$,

$$
\left|\int_{\psi_{2 k-1}} \operatorname{cs}_{2 k-1}(A)\right| \leq \sum_{j \in J}\left|\int_{\phi} e_{0, j} d e_{1, j} \cdots d e_{l_{j}, j}\right|,
$$

where the sum is over certain $e_{i, j} \in \mathcal{A}$ (because $\mathcal{A}$ is unital) with $e_{0, j} \cdots e_{l_{j}, j}=\prod_{m=1}^{M} a_{j_{m}} b_{j_{m}}$ for some $M$ with $k \leq M \leq 2 k-1$. The number of elements in $J$ is exponential in $k$. We obtain

$$
\left|\int_{\psi_{2 k-1}} \operatorname{cs}_{2 k-1}(A)\right| \leq \breve{C}_{f, s, n, \gamma}^{k} k !^{\gamma-1} \max \left(R^{2 k}, R^{4 k-2}\right) \operatorname{Tr}\left|(D-i)^{-s}\right|,
$$

for some number $\check{C}_{f, s, n, \gamma} \geq 1$. Similarly, we obtain a number $\hat{C}_{f, s, n, \gamma} \geq 1$ such that

$$
\left|\int_{\phi_{2 k}} F^{k}\right| \leq \hat{C}_{f, s, n, \gamma}^{k} k !^{\gamma-1} \max \left(R^{2 k}, R^{4 k}\right) \operatorname{Tr}\left|(D-i)^{-s}\right|,
$$

thereby proving the theorem.

This theorem has two important corollaries, for $f \in \mathcal{E}^{s, 1}$ (hence, for all $f \in \mathcal{E}^{s, \gamma}$ ) and for $f \in \mathcal{E}^{s, \gamma}, \gamma<1$

Corollary 34. Let $(\mathcal{A}, \mathcal{H}, D)$ be an s-summable spectral triple, let $f \in \mathcal{E}^{s, 1}$ and $V=\pi_{D}(A) \in \Omega_{D}^{1}(\mathcal{A})_{\text {sa. }}$. Then there exists a $\delta>0$ such that for all $t \in \mathbb{R}$ with $|t|<\delta$, we have

$$
\operatorname{Tr}(f(D+t V)-f(D))=\sum_{k=1}^{\infty}\left(\int_{\psi_{2 k-1}} \operatorname{cs}_{2 k-1}(t A)+\frac{1}{2 k} \int_{\phi_{2 k}} F_{t}^{k}\right),
$$

and the series converges absolutely. 
Proof. Write $V=\sum_{j=1}^{n} a_{j}\left[D, b_{j}\right]$. First take $C_{f, s, n, 1} \geq 1$ from Theorem 33 , define $R:=1 /\left(C_{f, s, n, 1}+1\right)$ such that $C_{f, s, n, 1} R<1$, and define $\delta:=\left(\frac{R}{\max _{j}\left\{\left\|a_{j}\right\|,\left\|b_{j}\right\|,\left\|\left[D, a_{j}\right]\right\|,\left\|\left[D, b_{j}\right]\right\|\right\}}\right)^{2}$. By writing

$$
t V=\sum_{j=1}^{n} \sqrt{|t|} a_{j}\left[D, \operatorname{sign}(t) \sqrt{|t|} b_{j}\right]
$$

the corollary follows.

Proof of Theorem 27. This follows from Theorem 33 by taking $\gamma<1$.

\section{Gauge invariance and the pairing with K-theory}

Since the spectral action is a spectral invariant, it is in particular invariant under conjugation of $D$ by a unitary in the algebra $\mathcal{A}$. More generally, in the presence of an inner fluctuation we find that the spectral action is invariant under the transformation

$$
D+V \mapsto u(D+V) u^{*}=D+V^{u} ; \quad V^{u}=u\left[D, u^{*}\right]+u V u^{*}
$$

This transformation also holds at the level of the universal forms, with a gauge transformation of the form $A \mapsto A^{u}=u d u^{*}+u A u^{*}$. Let us analyze the behavior of the Chern-Simons and YangMills terms appearing in Theorem 27 under this gauge transformation, and derive an interesting consequence for the pairing between the odd $(b, B)$-cocycle $\tilde{\psi}$ with the odd K-theory group of $\mathcal{A}$.

Lemma 35. The Yang-Mills terms $\int_{\phi_{2 k}} F^{k}$ with $F=d A+A^{2}$ are invariant under the gauge transformation $A \mapsto A^{u}$ for every $k \geq 1$.

Proof. Since the curvature of $A^{u}$ is simply given by $u F u^{*}$, the claim follows from Corollary 24)(i).

We are thus led to the conclusion that the Chern-Simons forms are gauge invariant as well. Indeed, arguing as in [11], since both $\operatorname{Tr} f(D+V)$ and the Yang-Mills terms are invariant under $V \mapsto V^{u}$, we find that, under the assumptions stated in Theorem 27 .

$$
\sum_{k=0}^{\infty} \int_{\psi_{2 k+1}} \operatorname{cs}_{2 k+1}\left(A^{u}\right)=\sum_{k=0}^{\infty} \int_{\psi_{2 k+1}} \operatorname{cs}_{2 k+1}(A) .
$$

Each individual Chern-Simons form behaves non-trivially under a gauge transformation. Nevertheless, it turns out that we can conclude, just as in [11], that the pairing of the whole $(b, B)$-cocycle with K-theory is trivial. Since the $(b, B)$-cocycle $\tilde{\psi}$ is given as an infinite sequence, we should first carefully study the analytical behavior of $\tilde{\psi}$. In fact, we should show that it is antire cyclic cocycle in the sense of [8] (see also [9, Section IV.7. $\alpha$ ]). For this purpose, we can without loss of generality assume that $\mathcal{A}$ is complete in the Banach algebra norm defined by $\|a\|_{1}:=\|a\|+\|[D, a]\|$, because $\left(\overline{\mathcal{A}}^{\|\cdot\|_{1}}, \mathcal{H}, D\right)$ is also a spectral triple, and the resulting $\tilde{\psi}_{2 k+1} \in \mathcal{C}^{2 k+1}\left(\overline{\mathcal{A}}^{\|\cdot\|_{1}}\right)$ is an extension of the one in $\mathcal{C}^{2 k+1}(\mathcal{A})$. Recall that for Banach algebras $\mathcal{A}$ an odd cochain such as $\tilde{\psi}$ is called entire if 
the power series $\sum_{k} \frac{(2 k+1) !}{k !}\left\|\tilde{\psi}_{2 k+1}\right\| z^{k}$ converges everywhere in $\mathbb{C}$. This is equivalent [9, Remark IV.7.7a,c] to the condition that for any bounded subset $\Sigma \subset \mathcal{A}$ there exists a constant $C_{\Sigma}$ so that

$$
\left|\tilde{\psi}_{2 k+1}\left(a_{0}, \ldots, a_{2 k+1}\right)\right| \leq \frac{C_{\Sigma}}{k !} \quad\left(\forall a_{j} \in \Sigma\right) .
$$

In our case it turns out that Lemma 7 implies the following growth condition, guaranteeing that indeed $\tilde{\psi}$ is entire.

Lemma 36. Fix $f \in \mathcal{E}^{s, \gamma}$ for $\gamma<1$ and equip $\mathcal{A}$ with the norm $\|a\|_{1}=\|a\|+\|[D, a]\|$. Then, for any bounded subset $\Sigma \subset \mathcal{A}$ there exists $C_{\Sigma}$ such that

$$
\left|\tilde{\psi}_{2 k+1}\left(a_{0}, \ldots, a_{2 k+1}\right)\right| \leq \frac{C_{\Sigma}}{k !}
$$

for all $a_{j} \in \Sigma$.

Proof. Assume that $\left\|a_{j}\right\|_{1} \leq R$ for all $a_{j} \in \Sigma$ so that both $\left\|a_{j}\right\|,\left\|\left[D, a_{j}\right]\right\| \leq R$. By definition of $\phi$, the expression $\psi_{2 k+1}\left(a_{0}, \ldots, a_{2 k+1}\right)$ is given by a linear combination of multiple operator integrals with arguments in $\{V \in \mathcal{B}(\mathcal{H}):\|V\| \leq R\}$ except for $a_{0}\left[D, a_{1}\right]$, which is bounded by $R^{2}$. By applying Lemma 7 , we obtain the estimate

$$
\left|\psi_{2 k+1}\left(a_{0}, \ldots, a_{2 k+1}\right)\right| \leq\left((2 k+1) \frac{C^{2 k+2}}{(2 k+1) !^{1-\gamma}}+(k+1) \frac{C^{2 k+3}}{(2 k+2) !^{1-\gamma}}\right) R^{2 k+2}\left\|(D-i)^{-1}\right\|_{s}^{s} .
$$

We recall from Proposition 19 that

$$
\tilde{\psi}_{2 k+1}=(-1)^{k} \frac{k !}{(2 k+1) !} \psi_{2 k+1}
$$

so that (34) in particular implies the lemma by use of, for instance, Stirling's approximation.

For $u \in M_{q}(\mathcal{A})$, define a pairing

$$
\langle u, \tilde{\psi}\rangle:=(2 \pi i)^{-1 / 2} \sum_{k=0}^{\infty}(-1)^{k} k ! \tilde{\psi}_{2 k+1}^{q}\left(u^{*}, u, \ldots, u^{*}, u\right),
$$

where $\tilde{\psi}_{2 k+1}^{q}:=\operatorname{Tr} \# \tilde{\psi}_{2 k+1}:\left(\mu_{0} \otimes a_{0}, \ldots, \mu_{2 k+1} \otimes a_{2 k+1}\right) \mapsto \operatorname{Tr}\left(\mu_{0} \cdots \mu_{2 k+1}\right) \tilde{\psi}_{2 k+1}\left(a_{0}, \ldots, a_{2 k+1}\right)$ for $\mu_{0}, \ldots, \mu_{2 k+1} \in M_{q}(\mathbb{C})$ and $a_{0}, \ldots, a_{2 k+1} \in \mathcal{A}$. Since $\tilde{\psi}$ is a $(b, B)$-cocycle, it follows from [9. Corollary IV.7.27] (see also [9, Sections III.3 and IV.7]) that this pairing only depends on the class of $u$ in $K_{1}(\mathcal{A})$.

Theorem 37. Let $f \in \mathcal{E}^{s, \gamma}$ for $\gamma<1$. Then the pairing of the odd entire cyclic cocycle $\tilde{\psi}$ with $K_{1}(\mathcal{A})$ is trivial, i.e.

$$
\langle u, \tilde{\psi}\rangle=0
$$

for all unitary $u \in M_{q}(\mathcal{A})$. 
Proof. Apply Theorem 27 to a bigger spectral triple, namely $\left(\mathcal{A}^{q}, \mathcal{H}^{q}, D^{q}\right):=\left(M_{q}(\mathbb{C}) \otimes \mathcal{A}, \mathbb{C}^{q} \otimes\right.$ $\left.\mathcal{H}, I_{q} \otimes D\right)$. Take $A=u^{*} d u$ for $u$ unitary in $M_{q}(\mathcal{A})=M_{q}(\mathbb{C}) \otimes \mathcal{A}$. Clearly, then $V=u^{*}\left[D^{q}, u\right]$, and because the multiple operator integral behaves naturally with respect to tensor products, we obtain

$$
\operatorname{Tr}\left(f\left(D^{q}+u^{*}\left[D^{q}, u\right]\right)-f\left(D^{q}\right)\right)=\sum_{k=0}^{\infty}\left(\int_{\psi_{2 k+1}^{q}} \operatorname{cs}_{2 k+1}\left(u^{*} d u\right)+\frac{1}{2 k+2} \int_{\phi_{2 k+2}^{q}} F^{k+1}\right),
$$

where $F=d\left(u^{*} d u\right)+\left(u^{*} d u\right)^{2}=0$. Also notice that the left-hand side equals $\operatorname{Tr}\left(f\left(u^{*} D^{q} u\right)-\right.$ $\left.f\left(D^{q}\right)\right)=0$. Therefore,

$$
\sum_{k=0}^{\infty} \int_{\psi_{2 k+1}^{q}} \operatorname{cs}_{2 k+1}\left(u^{*} d u\right)=0
$$

From the definition of the Chern-Simons form (Definition 25) and the fact that $F_{t}=t d A+t^{2} A^{2}=$ $\left(t-t^{2}\right) d A+t^{2} F=\left(t-t^{2}\right) d u^{*} d u$ we find that

$$
\operatorname{cs}_{2 k+1}\left(u^{*} d u\right)=\int_{0}^{1} d t\left(t-t^{2}\right)^{k} u^{*} d u d u^{*} d u \cdots d u^{*} d u
$$

so that by a straightforward integration we may conclude that

$$
\int_{\psi_{2 k+1}^{q}} \operatorname{cs}_{2 k+1}\left(u^{*} d u\right)=\frac{k !^{2}}{(2 k+1) !} \psi_{2 k+1}^{q}\left(u^{*}, u, \ldots, u^{*}, u\right) .
$$

Combining this with (35), (36) and (37), the theorem follows.

Acknowledgements We would like to thank Steven Lord and Fedor Sukochev for their generous hospitality during a visit in Summer 2019. We also thank them, the members in their research group, as well as the other participants in the workshop "Noncommutative Calculus and the Spectral Action" at UNSW in August 2019 for fruitful discussions. We also thank Alain Connes and Anna Skripka for useful comments. Research supported by NWO Physics Projectruimte (68091-101).

\section{References}

[1] N. A. Azamov, A. L. Carey, P. G. Dodds, and F. A. Sukochev. Operator integrals, spectral shift, and spectral flow. Canad. J. Math. (2) 61 (2009), 241-263.

[2] A. H. Chamseddine and A. Connes. Universal formula for noncommutative geometry actions: Unifications of gravity and the Standard Model. Phys. Rev. Lett. 77 (1996), 4868-4871.

[3] A. H. Chamseddine and A. Connes. The spectral action principle. Commun. Math. Phys. 186 (1997), 731-750.

[4] A. H. Chamseddine, A. Connes, and M. Marcolli. Gravity and the Standard Model with neutrino mixing. Adv. Theor. Math. Phys. (6) 11 (2007) 991-1089. 
[5] Y. Choquet-Bruhat and C. DeWitt-Morette. Analysis, manifolds and physics. Part II. NorthHolland Publishing Co., Amsterdam, 1989. 92 applications.

[6] Y. Choquet-Bruhat, C. DeWitt-Morette, and M. Dillard-Bleick. Analysis, manifolds and physics. North-Holland Publishing Co., Amsterdam-New York-Oxford, 1977.

[7] A. Connes. Non-commutative differential geometry. Publ. Math. IHES 62 (1985) 257-360.

[8] A. Connes. Entire cyclic cohomology of Banach algebras and characters of $\theta$-summable Fredholm modules. K-Theory 1 (1988) 519-548.

[9] A. Connes. Noncommutative Geometry. Academic Press, San Diego, 1994.

[10] A. Connes. Gravity coupled with matter and the foundation of non-commutative geometry. Commun. Math. Phys. 182 (1996) 155-176.

[11] A. Connes and A. H. Chamseddine. Inner fluctuations of the spectral action. J. Geom. Phys. 57 (2006) 1-21.

[12] A. Chattopadhyay and A. Skripka. Trace formulas for relative Schatten class perturbations. J. Funct. Anal. (12) 274 (2018), 3377-3410.

[13] A. Connes and M. Marcolli. Noncommutative Geometry, Quantum Fields and Motives. AMS, Providence, 2008.

[14] A. Carey and J. Phillips. Unbounded Fredholm modules and spectral flow Can. J. Math. (4) 50 (1998), 673-718.

[15] E. Getzler and A. Szenes. On the Chern character of a theta-summable Fredholm module. J. Funct. Anal. (2) 84 (1989) 343-357.

[16] N. Higson. The residue index theorem of Connes and Moscovici. In Surveys in noncommutative geometry, volume 6 of Clay Math. Proc., pages 71-126. Amer. Math. Soc., Providence, RI, 2006.

[17] T. D. H. van Nuland and A. Skripka. Spectral shift for relative Schatten class perturbations. arXiv:2102.00090 [math.FA].

[18] M. Nakahara. Geometry, Topology and Physics. IOP Publishing, 1990.

[19] S. Paycha. (Second) Quantised resolvents and regularised traces. J. Geom. Phys. (5) 57 (2007) 1345-1369.

[20] D. Potapov, A. Skripka, and F. Sukochev. Spectral shift function of higher order. Invent. Math. (3) 193 (2013), 501-538.

[21] D. Quillen. Chern-Simons forms and cyclic cohomology. In The interface of mathematics and particle physics (Oxford, 1988), volume 24 of Inst. Math. Appl. Conf. Ser. New Ser., pages 117-134. Oxford Univ. Press, New York, 1990.

[22] A. Skripka. Asymptotic expansions for trace functionals. J. Funct. Anal. (5) 266 (2014) 28452866. 
[23] A. Skripka and A. Tomskova. Multilinear Operator Integrals: Theory and Applications. Lecture Notes in Math. 2250, Springer International Publishing, 2019, XI+192 pp.

[24] W. D. van Suijlekom. Perturbations and operator trace functions. J. Funct. Anal. (8) 260 (2011) 2483-2496.

[25] W. D. van Suijlekom. Noncommutative Geometry and Particle Physics. Springer, 2015. 